\title{
Periodic Bifurcations in Descendant Trees of Finite $p$-Groups
}

\author{
Daniel C. Mayer \\ Naglergasse 53, 8010 Graz, Austria \\ Email: algebraic.number.theory@algebra.at
}

Received 20 February 2015; accepted 13 March 2015; published 23 March 2015

Copyright (C) 2015 by author and Scientific Research Publishing Inc.

This work is licensed under the Creative Commons Attribution International License (CC BY).

http://creativecommons.org/licenses/by/4.0/

c) (i) Open Access

\begin{abstract}
Theoretical background and an implementation of the $p$-group generation algorithm by Newman and 0 'Brien are used to provide computational evidence of a new type of periodically repeating patterns in pruned descendant trees of finite $p$-groups.
\end{abstract}

\section{Keywords}

Finite $p$-Group, Central Series, Descendant Tree, Pro-p Group, Coclass Tree, $p$-Covering Group, Nuclear Rank, Multifurcation, Coclass Graph, Parametrized Presentation, Commutator Calculus, Schur $\sigma$-Group

\section{Introduction}

In $\S \S 2-11$, we present an exposition of facts concerning the mathematical structure which forms the central idea of this article: descendant trees of finite $p$-groups. Their computational construction is recalled in $\S \S 12-20$ on the $p$-group generation algorithm. Recently periodic patterns have been discovered in descendant trees with promising arithmetical applications form the topic of the final $\S 21$ and the coronation of the entire work.

\section{The Structure: Descendant Trees}

In mathematics, specifically group theory, a descendant tree is a hierarchical structure for visualizing parentdescendant relations ( $\$ \S 4$ and 6) between isomorphism classes of finite groups of prime power order $p^{n}$, for a fixed prime number $p$ and varying integer exponents $n \geq 0$. Such groups are briefly called finite $p$-groups. The vertices of a descendant tree are isomorphism classes of finite $p$-groups.

Additionally to their order $p^{n}$, finite $p$-groups possess two further related invariants, the nilpotency class $c$ and the coclass $r:=n-c(\S \S 5$ and 8$)$. It turned out that descendant trees of a particular kind, the so-called 
pruned coclass trees whose infinitely many vertices share a common coclass $r$, reveal a repeating finite pattern (§7). These two crucial properties of finiteness and periodicity, which have been proved independently by du Sautoy [1] and by Eick and Leedham-Green [2], admit a characterization of all members of the tree by finitely many parametrized presentations ( $\S 10$ and 21 ). Consequently, descendant trees play a fundamental role in the classification of finite $p$-groups. By means of kernels and targets of Artin transfer homomorphisms [3], descendant trees can be endowed with additional structure [4]-[6], which recently turned out to be decisive for arithmetical applications in class field theory, in particular, for determining the exact length of $p$-class towers [7].

An important question is how the descendant tree $\mathcal{T}(R)$ can actually be constructed for an assigned starting group which is taken as the root $R$ of the tree. Sections $\S \S 13-19$ are devoted to recall a minimum of the necessary background concerning the $p$-group generation algorithm by Newman [8] and O'Brien [9] [10], which is a recursive process for constructing the descendant tree of a foregiven finite $p$-group playing the role of the tree root. This algorithm is now implemented in the ANUPQ-package [11] of the computational algebra systems GAP [12] and MAGMA [13].

As a final highlight in $\S 21$, whose formulation requires an understanding of all the preceding sections, this article concludes with brand-new discoveries of an unknown, and up to now unproved, kind of repeating infinite patterns called periodic bifurcations, which appeared in extensive computational constructions of descendant trees of certain finite 2-groups, resp. 3-groups, $G$ with abelianization $G / G^{\prime}$ of type $(2,2,2)$, resp. $(3,3)$, and have immediate applications in algebraic number theory and class field theory.

\section{Historical Remarks on Bifurcation}

Since computer aided classifications of finite $p$-groups go back to 1975 , fourty years ago, there arises the question why periodic bifurcations did not show up in the earlier literature already. At the first sight, this fact seems incomprehensible, because the smallest two 3-groups which reveal the phenomenon of periodic bifurcations with modest complexity were well known to both, Ascione, Havas and Leedham-Green [14] and Nebelung [15]. Their SmallGroups identifiers are $\langle 729,49\rangle$ and $\langle 729,54\rangle$ (see $\$ 9$ and [16] [17]). Due to the lack of systematic identifiers in 1977, they were called the non-CF groups $Q$ and $U$ in ([14], Table 1, p. 265, and Table 2, p. 266), since their lower central series $\left(\gamma_{j}(G)\right)$ has a non-cyclic factor $\gamma_{3}(G) / \gamma_{4}(G)$ of type $(3,3)$. Similarly, there was no SmallGroups Database yet in 1989 , whence the two groups were designated by $G_{0}^{5,6}(0,-1,0,1)$ and $G_{0}^{5,6}(0,0,0,1)$ in ([15], Satz 6.14, p. 208).

So Ascione and Nebelung were both standing in front of the door to a realm of uncharted waters. The reason why they did not enter this door was the sharp definition of their project targets. A bifurcation is the special case of a 2-fold multifurcation $(\S 8)$ : At a vertex $G$ of coclass $\operatorname{cc}(G)=r$ with nuclear rank $v(G)=2$, the descendant tree $\mathcal{T}(G)$ forks into a regular component of the same coclass $\mathcal{T}^{r}(G)$ and an irregular component of the next coclass $\mathcal{T}^{r+1}(G)$.

Ascione's thesis subject [18] [19] in 1979 was to investigate two-generated 3-groups $G$ of second maximal class, that is, of coclass $\operatorname{cc}(G)=2$. Consequently, she studied the regular tree $\mathcal{T}^{2}(G)$ for $G \in\{Q, U\}$ and did not touch the irregular component $\mathcal{T}^{3}(G)$ whose members are not of second maximal class.

The goal of Nebelung's dissertation [15] in 1989 was the classification of metabelian 3-groups $G$ with $G / G^{\prime}$ of type $(3,3)$. Therefore she focused on the metabelian skeleton $\mathcal{T}_{*}^{2}(G)$ of the regular coclass tree $\mathcal{T}^{2}(G)$ for $G \in\{Q, U\} \quad$ (a special case of a pruned coclass tree, see $\S 7$ ) and omitted the irregular component $\mathcal{T}^{3}(G)$ whose members are entirely non-metabelian of derived length 3.

\section{Definitions and Terminology}

According to Newman ([20], 2, pp. 52-53), there exist several distinct definitions of the parent $\pi(G)$ of a finite $p$-group $G$. The common principle is to form the quotient $\pi(G):=G / N$ of $G$ by a suitable normal subgroup $N \unlhd G$ which can be either

$[(\mathrm{P})]$

1) the centre $N=\zeta_{1}(G)$ of $G$, whence $\pi(G)=G / \zeta_{1}(G)$ is called central quotient of $G$ or

2) the last non-trivial term $N=\gamma_{c}(G)$ of the lower central series of $G$, where $c$ denotes the nilpotency class of $G$ or

3) the last non-trivial term $N=P_{c-1}(G)$ of the lower exponent- $p$ central series of $G$, where $c$ denotes 
the exponent- $p$ class of $G$ or

4) the last non-trivial term $N=G^{(d-1)}$ of the derived series of $G$, where $d$ denotes the derived length of $G$.

In each case, $G$ is called an immediate descendant of $\pi(G)$ and a directed edge of the tree is defined either by $G \rightarrow \pi(G)$ in the direction of the canonical projection $\pi: G \rightarrow \pi(G)$ onto the quotient $\pi(G)=G / N$ or by $\pi(G) \rightarrow G$ in the opposite direction, which is more usual for descendant trees. The former convention is adopted by Leedham-Green and Newman ([21], 2, pp. 194-195), by du Sautoy and Segal ([22], 7, p. 280), by Leedham-Green and McKay ([23], Dfn.8.4.1, p. 166), and by Eick, Leedham-Green, Newman and O'Brien ([24], 1). The latter definition is used by Newman ([20], 2, pp. 52-53), by Newman and O'Brien ([25], 1, p. 131), by du Sautoy ([1], 1, p. 67), by Dietrich, Eick and Feichtenschlager ([26], 2, p. 46) and by Eick and Leedham-Green ([2], 1, p. 275).

In the following, the direction of the canonical projections is selected for all edges. Then, more generally, a vertex $R$ is a descendant of a vertex $P$, and $P$ is an ancestor of $R$, if either $R$ is equal to $P$ or there is a path

$$
R=Q_{0} \rightarrow Q_{1} \rightarrow \cdots \rightarrow Q_{m-1} \rightarrow Q_{m}=P, \text { with } m \geq 1
$$

of directed edges from $R$ to $P$. The vertices forming the path necessarily coincide with the iterated parents $Q_{j}=\pi^{j}(R)$ of $R$, with $0 \leq j \leq m$ :

$$
R=\pi^{0}(R) \rightarrow \pi^{1}(R) \rightarrow \cdots \rightarrow \pi^{m-1}(R) \rightarrow \pi^{m}(R)=P \text {, with } m \geq 1
$$

In the most important special case (P2) of parents defined as last non-trivial lower central quotients, they can also be viewed as the successive quotients $R / \gamma_{c+1-j}(R)$ of class $c-j$ of $R$ when the nilpotency class of $R$ is given by $c \geq m$ :

$$
R \simeq R / \gamma_{c+1}(R) \rightarrow R / \gamma_{C}(R) \rightarrow \cdots \rightarrow R / \gamma_{c+2-m}(R) \rightarrow R / \gamma_{c+1-m}(R) \simeq P
$$

with $c \geq m \geq 1$.

Generally, the descendant tree $\mathcal{T}(G)$ of a vertex $G$ is the subtree of all descendants of $G$, starting at the root $G$. The maximal possible descendant tree $\mathcal{T}(1)$ of the trivial group 1 contains all finite $p$-groups and is somewhat exceptional, since, for any parent definition (P1 - P4), the trivial group 1 has infinitely many abelian $p$-groups as its immediate descendants. The parent definitions (P2 - P3) have the advantage that any non-trivial finite $p$-group (of order divisible by $p$ ) possesses only finitely many immediate descendants.

\section{Pro- $p$ Groups and Coclass Trees}

For a sound understanding of coclass trees as a particular instance of descendant trees, it is necessary to summarize some facts concerning infinite topological pro- $p$ groups. The members $\gamma_{j}(S)$, with $j \geq 1$, of the lower central series of a pro- $p$ group $S$ are open and closed subgroups of finite index, and therefore the corresponding quotients $S / \gamma_{j}(S)$ are finite $p$-groups. The pro- $p$ group $S$ is said to be of coclass $\operatorname{cc}(S):=r$ when the limit $r=\lim _{j \rightarrow \infty} \operatorname{cc}\left(S / \gamma_{j}(S)\right)$ of the coclass of the successive quotients exists and is finite. An infinite pro- $p$ group $S$ of coclass $r$ is a $p$-adic pre-space group ([23], Dfn.7.4.11, p. 147), since it has a normal subgroup $T$, the translation group, which is a free module over the ring $\mathbb{Z}_{p}$ of $p$-adic integers of uniquely determined rank $d$, the dimension, such that the quotient $P=S / T$ is a finite $p$-group, the point group, which acts on $T$ uniserially. The dimension is given by

$$
d=(p-1) p^{s}, \quad \text { with some } 0 \leq s<r
$$

A central finiteness result for infinite pro- $p$ groups of coclass $r$ is provided by the so-called Theorem $\mathrm{D}$, which is one of the five Coclass Theorems proved in 1994 independently by Shalev [27] and by Leedham-Green ([28], Thm.7.7, p. 66), and conjectured in 1980 already by Leedham-Green and Newman ([21], 2, pp. 194196). Theorem $\mathrm{D}$ asserts that there are only finitely many isomorphism classes of infinite pro- $p$ groups of coclass $r$, for any fixed prime $p$ and any fixed non-negative integer $r$. As a consequence, if $S$ is an infinite pro- $p$ group of coclass $r$, then there exists a minimal integer $i \geq 1$ such that the following three conditions are satisfied for any integer $j \geq i$. 
- $\operatorname{cc}\left(S / \gamma_{j}(S)\right)=r$

- $S / \gamma_{j}(S)$ is not a lower central quotient of any infinite pro- $p$ group of coclass $r$ which is not isomorphic to $S$;

- $\gamma_{j}(S) / \gamma_{j+1}(S)$ is cyclic of order $p$.

The descendant tree $\mathcal{T}(R)$, with respect to the parent definition (P2), of the root $R=S / \gamma_{i}(S)$ with minimal $i$ is called the coclass tree $\mathcal{T}(S)$ of $S$ and its unique maximal infinite (reverse-directed) path

$$
R=S / \gamma_{i}(S) \leftarrow S / \gamma_{i+1}(S) \leftarrow S / \gamma_{i+2}(S) \leftarrow \cdots
$$

is called the mainline (or trunk) of the tree.

\section{Tree Diagram}

Further terminology, used in diagrams visualizing finite parts of descendant trees, is explained in Figure 1 by means of an artificial abstract tree. On the left hand side, a level indicates the basic top-down design of a descendant tree. For concrete trees, such as those in Figure 2, resp. Figure 3, etc., the level is usually replaced by a scale of orders increasing from the top to the bottom. A vertex is capable (or extendable) if it has at least one immediate descendant, otherwise it is terminal (or a leaf). Vertices sharing a common parent are called siblings.

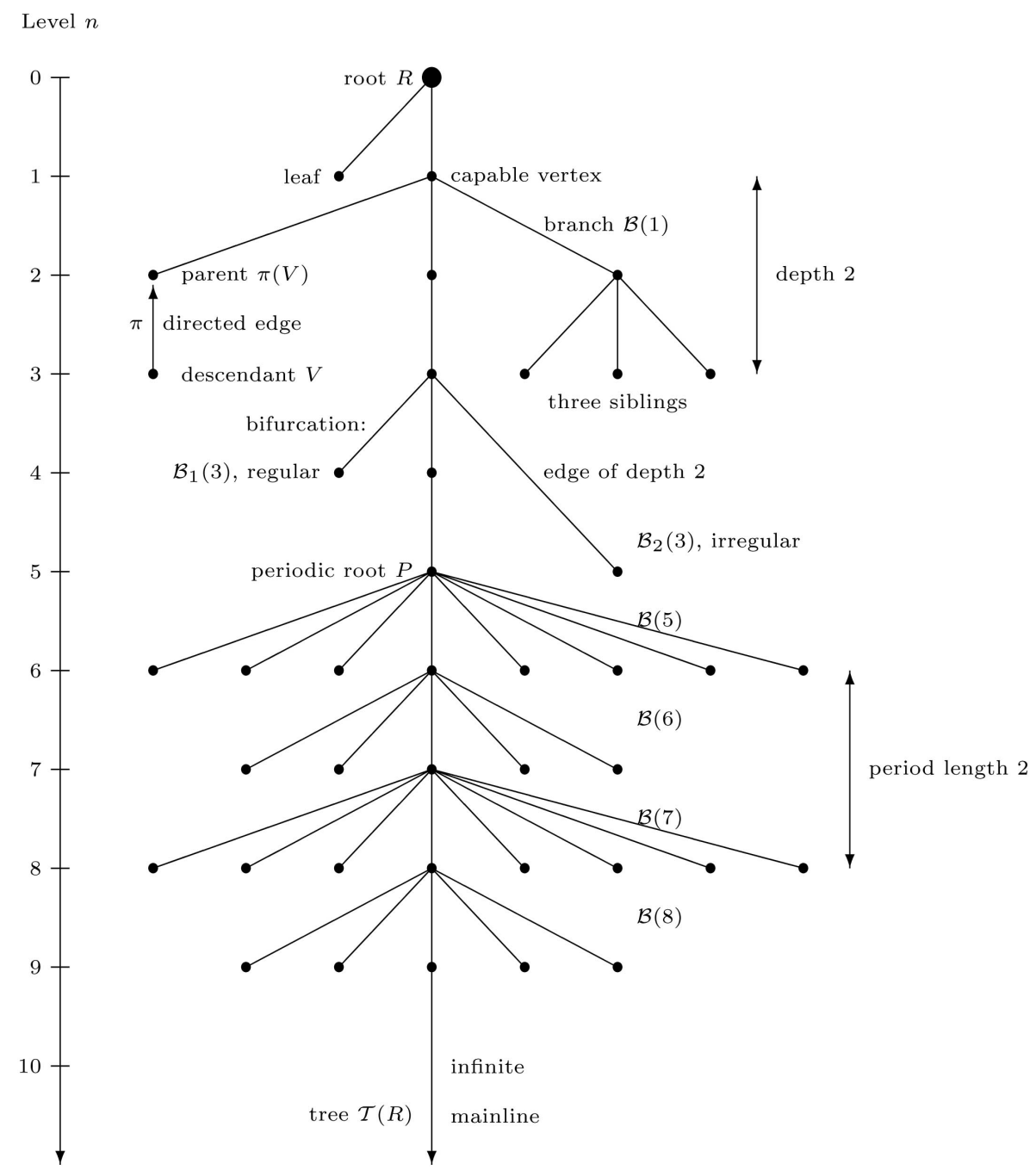

Figure 1. Terminology for descendant trees. 


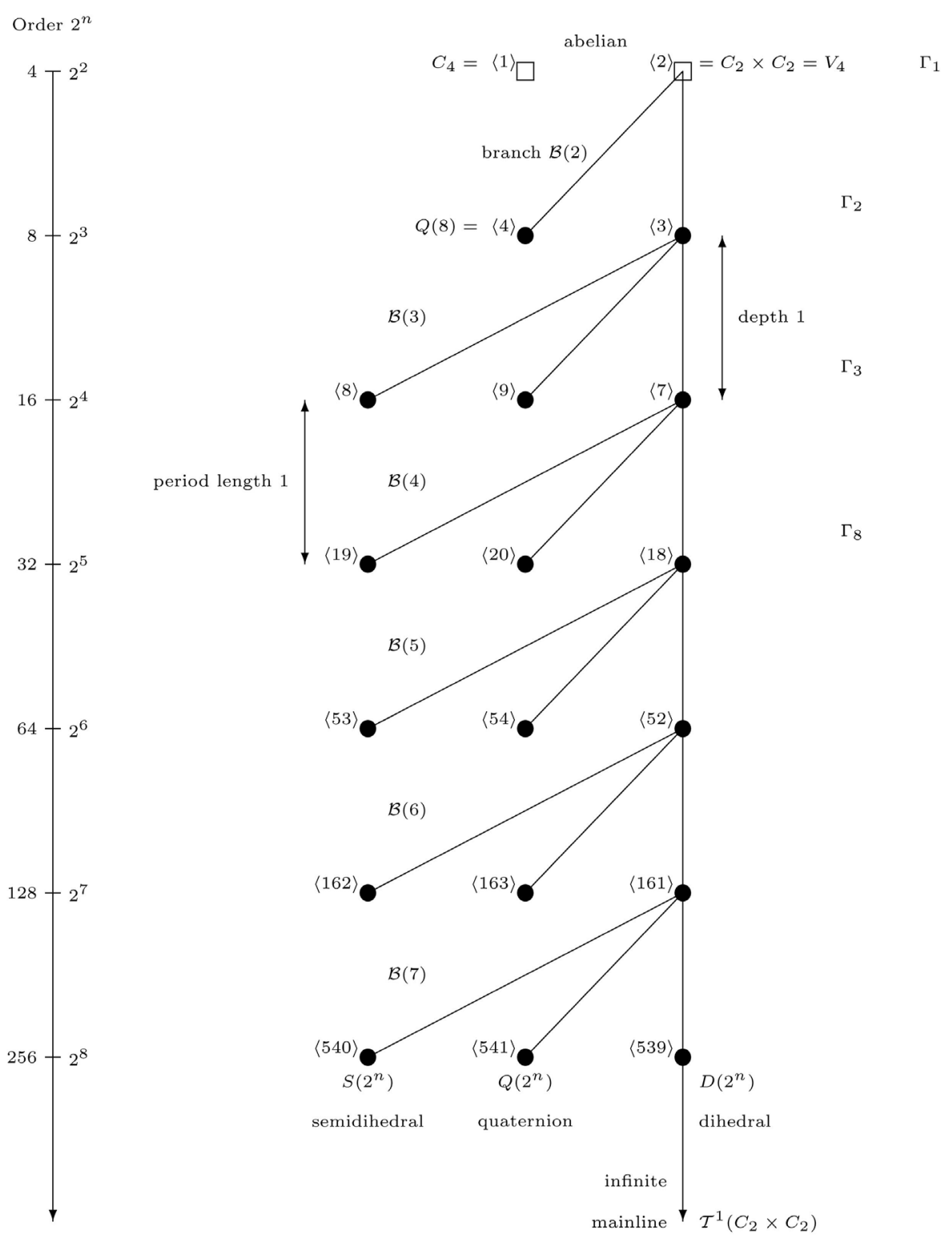

Figure 2. 2-groups of coclass 1.

If the descendant tree is a coclass tree $\mathcal{T}(R)$ with root $R=R_{0}$ and with mainline vertices $\left(R_{n}\right)_{n \geq 0}$ labelled according to the level $n$, then the finite subtree defined as the difference set

$$
\mathcal{B}(n):=\mathcal{T}\left(R_{n}\right) \backslash \mathcal{T}\left(R_{n+1}\right)
$$

is called the $n$th branch (or twig) of the tree or also the branch $\mathcal{B}\left(R_{n}\right)$ with root $R_{n}$, for any $n \geq 0$. The depth of a branch is the maximal length of the paths connecting its vertices with its root.

Figure 1 shows a descendant tree whose branches $\mathcal{B}(2), \mathcal{B}(4)$ both have depth 0 , and $\mathcal{B}(5) \simeq \mathcal{B}(7)$, resp. $\mathcal{B}(6) \simeq \mathcal{B}(8)$, are isomorphic as trees.

If all vertices of depth bigger than a given integer $k \geq 0$ are removed from branch $\mathcal{B}(n)$, then we obtain the (depth-)pruned branch $\mathcal{B}_{k}(n)$. Correspondingly, the pruned coclass tree $\mathcal{T}_{k}(R)$, resp. the entire coclass tree $\mathcal{T}(R)$, consists of the infinite sequence of its pruned branches $\left(\mathcal{B}_{k}(n)\right)_{n \geq 0}$, resp. branches $(\mathcal{B}(n))_{n \geq 0}$, connected by the mainline, whose vertices $R_{n}$ are called infinitely capable. 


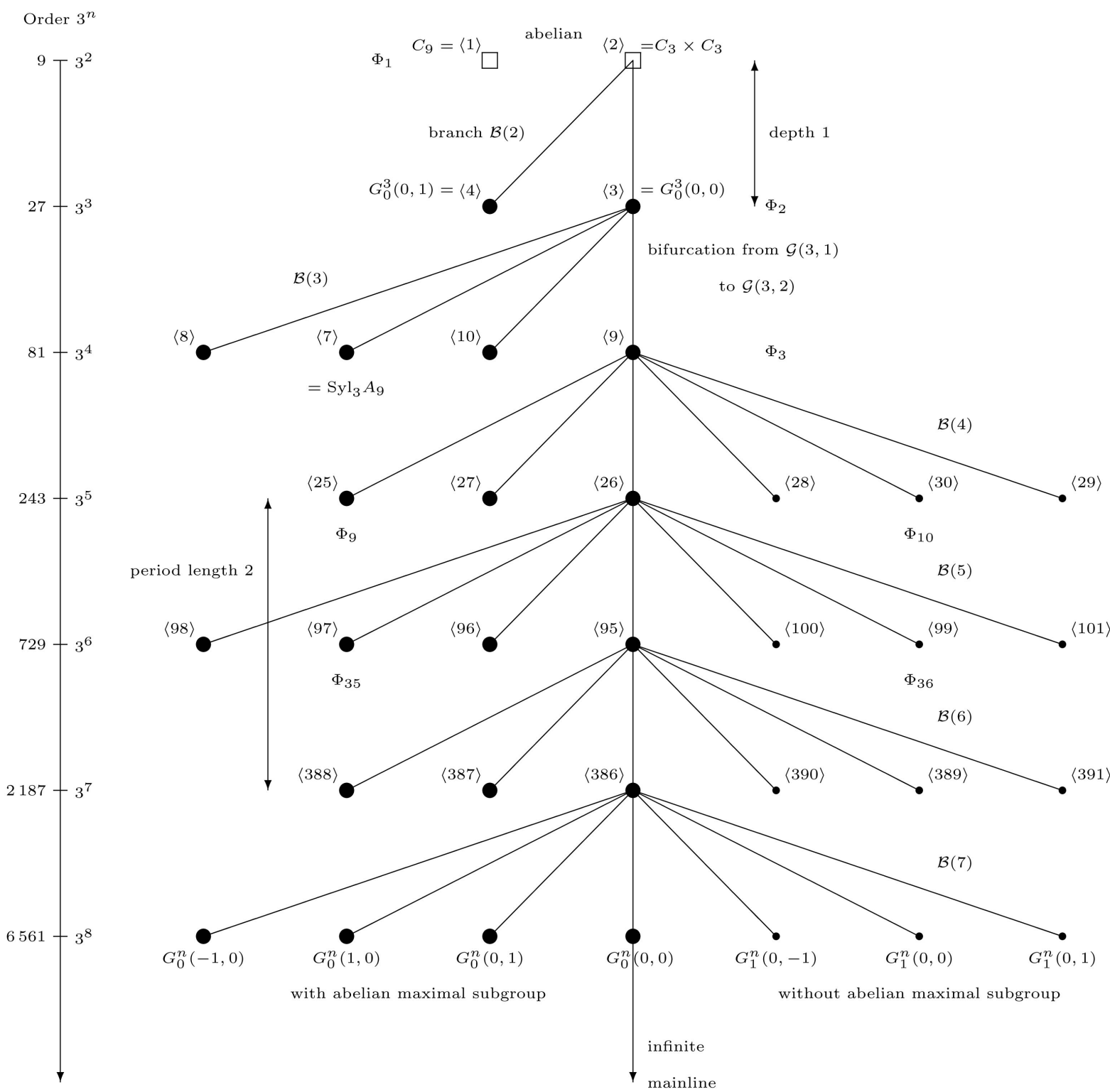

Figure 3. 3-groups of coclass 1.

\section{Virtual Periodicity}

The periodicity of branches of depth-pruned coclass trees has been proved with analytic methods using zeta functions ([22], 7, Thm.15, p. 280) of groups by du Sautoy ([1], Thm.1.11, p. 68, and Thm.8.3, p. 103), and with algebraic techniques using cohomology groups by Eick and Leedham-Green [2]. The former methods admit the qualitative insight of ultimate virtual periodicity, the latter techniques determine the quantitative structure.

Theorem 7.1 For any infinite pro- $p$ group $S$ of coclass $r \geq 1$ and dimension $d$, and for any given depth $k \geq 1$, there exists an effective minimal lower bound $f(k) \geq 1$, where periodicity of length $d$ of depth$k$ pruned branches of the coclass tree $\mathcal{T}(S)$ sets in, that is, there exist graph isomorphisms

$$
\mathcal{B}_{k}(n+d) \simeq \mathcal{B}_{k}(n), \text { for all } n \geq f(k)
$$

Proof. The graph isomorphisms of depth- $k$ pruned banches with roots of sufficiently large order $n \geq f(k)$ are derived with cohomological methods in ([2], Thm.6, p. 277, Thm.9, p. 278) and the effective lower bound $f(k)$ for the branch root orders is established in ([2], Thm.29, p. 287). 
This central result can be expressed ostensively: When we look at a coclass tree through a pair of blinkers and ignore a finite number of pre-periodic branches at the top, then we shall see a repeating finite pattern (ultimate periodicity). However, if we take wider blinkers the pre-periodic initial section may become longer (virtual periodicity).

The vertex $P=R_{f(k)}$ is called the periodic root of the pruned coclass tree, for a fixed value of the depth $k$. See Figure 1.

\section{Multifurcation and Coclass Graphs}

Assume that parents of finite $p$-groups are defined as last non-trivial lower central quotients (P2). For a $p$-group $G$ of coclass $\operatorname{cc}(G)=r$, we can distinguish its (entire) descendant tree $\mathcal{T}(G)$ and its coclass- $r$ descendant tree $\mathcal{T}^{r}(G)$, the subtree consisting of descendants of coclass $r$ only. The group $G$ is coclass-settled if $\mathcal{T}(G)=\mathcal{T}^{r}(G)$.

The nuclear rank $v(G)$ of $G$ (see $\S 14)$ in the theory of the $p$-group generation algorithm by Newman [8] and O'Brien [9] provides the following criteria.

- $G$ is terminal, and thus trivially coclass-settled, if and only if $v(G)=0$;

- If $v(G)=1$, then $G$ is capable, but it remains unknown whether $G$ is coclass-settled;

- If $v(G)=m \geq 2$, then $G$ is capable and certainly not coclass-settled.

In the last case, a more precise assertion is possible: If $G$ has coclass $r$ and nuclear $\operatorname{rank} v(G)=m \geq 2$, then it gives rise to an $m$-fold multifurcation into a regular coclass- $r$ descendant tree $\mathcal{T}^{r}(G)$ and $m-1$ irregular descendant graphs $\mathcal{T}^{r+j}(G)$ of coclass $r+j$, for $1 \leq j \leq m-1$. Consequently, the descendant tree of $G$ is the disjoint union

$$
\mathcal{T}(G)=\dot{\cup}_{j=0}^{m-1} \mathcal{T}^{r+j}(G)
$$

Multifurcation is correlated with different orders of the last non-trivial lower central of immediate descendants. Since the nilpotency class increases exactly by a unit, $c=\operatorname{cl}(Q)=\operatorname{cl}(P)+1$, from a parent $P=\pi(Q)$ to any immediate descendant $Q$, the coclass remains stable, $r=\operatorname{cc}(Q)=\operatorname{cc}(P)$, if $\left|\gamma_{c}(Q)\right|=p$. In this case, $Q$ is a regular immediate descendant with directed edge $P \leftarrow Q$ of depth 1 , as usual. However, the coclass increases by $m-1$, if $\left|\gamma_{c}(Q)\right|=p^{m}$ with $m \geq 2$. Then $Q$ is called an irregular immediate descendant with directed edge of depth $m$.

If the condition of depth (or step size) 1 is imposed on all directed edges, then the maximal descendant tree $\mathcal{T}(1)$ of the trivial group 1 splits into a countably infinite disjoint union

$$
\mathcal{T}(1)=\dot{\cup}_{r=0}^{\infty} \mathcal{G}(p, r)
$$

of directed coclass graphs $\mathcal{G}(p, r)$, which are rather forests than trees. More precisely, the above mentioned Coclass Theorems imply that

$$
\mathcal{G}(p, r)=\left(\dot{\cup}_{i} \mathcal{T}\left(S_{i}\right)\right) \dot{\cup} \mathcal{G}_{0}(p, r)
$$

is the disjoint union of finitely many coclass trees $\mathcal{T}\left(S_{i}\right)$ of pairwise non-isomorphic infinite pro- $p$ groups $S_{i}$ of coclass $r$ (Theorem D) and a finite subgraph $\mathcal{G}_{0}(p, r)$ of sporadic groups lying outside of any coclass tree.

\section{Identifiers}

The SmallGroups Library identifiers of finite groups, in particular $p$-groups, given in the form

$$
\langle\text { order, counting number }
$$

in the following concrete examples of descendant trees, are due to Besche, Eick and O'Brien [16] [17]. When the group orders are given in a scale on the left hand side as in Figure 2 and Figure 3, the identifiers are briefly denoted by 


$$
\text { 〈counting number> }
$$

Depending on the prime $p$, there is an upper bound on the order of groups for which a SmallGroup identifier exists, e.g. $512=2^{9}$ for $p=2$, and $2187=3^{7}$ for $p=3$. For groups of bigger orders, a notation with generalized identifiers resembling the descendant structure is employed: a regular immediate descendant, connected by an edge of depth 1 with its parent $P$, is denoted by

$$
P-\# 1 \text {; counting number }
$$

and an irregular immediate descendant, connected by an edge of depth $d \geq 2$ with its parent $P$, is denoted by

$$
P-\# d \text {; counting number }
$$

The ANUPQ package [11] containing the implementation of the p-group generation algorithm uses this notation, which goes back to Ascione in 1979 [18].

\section{Concrete Examples of Trees}

In all examples, the underlying parent definition (P2) corresponds to the usual lower central series. Occasional differences to the parent definition (P3) with respect to the lower exponent- $p$ central series are pointed out.

\subsection{Coclass 0}

The coclass graph

$$
\mathcal{G}(p, 0)=\mathcal{G}_{0}(p, 0)
$$

of finite $p$-groups of coclass 0 does not contain a coclass tree and consists of the trivial group 1 and the cyclic group $C_{p}$ of order $p$, which is a leaf (however, it is capable with respect to the lower exponent- $p$ central series). For $p=2$ the SmallGroup identifier of $C_{p}$ is $\langle 2,1\rangle$, for $p=3$ it is $\langle 3,1\rangle$.

\subsection{Coclass 1}

The coclass graph

$$
\mathcal{G}(p, 1)=\mathcal{T}^{1}(R) \dot{\cup} \mathcal{G}_{0}(p, 1)
$$

of finite $p$-groups of coclass 1 consists of the unique coclass tree with root $R=C_{p} \times C_{p}$, the elementary abelian $p$-group of rank 2 , and a single isolated vertex (a terminal orphan without proper parent in the same coclass graph, since the directed edge to the trivial group 1 has depth 2), the cyclic group $C_{p^{2}}$ of order $p^{2}$ in the sporadic part $\mathcal{G}_{0}(p, 1)$ (however, this group is capable with respect to the lower exponent- $p$ central series). The tree $\mathcal{T}^{1}(R)=\mathcal{T}^{1}\left(S_{1}\right)$ is the coclass tree of the unique infinite pro- $p$ group $S_{1}$ of coclass 1 .

For $p=2$, resp. $p=3$, the SmallGroup identifier of the root $R$ is $\langle 4,2\rangle$, resp. $\langle 9,2\rangle$, and a tree diagram of the coclass graph from branch $\mathcal{B}(2)$ up to branch $\mathcal{B}(7)$ (counted with respect to the $p$-logarithm of the order of the branch root) is drawn in Figure 2, resp. Figure 3, where all groups of order at least $p^{3}$ are metabelian, that is non-abelian with derived length 2 (vertices represented by black discs in contrast to contour squares indicating abelian groups). In Figure 3, smaller black discs denote metabelian 3-groups where even the maximal subgroups are non-abelian, a feature which does not occur for the metabelian 2-groups in Figure 2, since they all possess an abelian subgroup of index $p$ (usually exactly one). The coclass tree of $\mathcal{G}(2,1)$, resp. $\mathcal{G}(3,1)$, has periodic root $\langle 8,3\rangle$ and period of length 1 starting with branch $\mathcal{B}(3)$, resp. periodic root $\langle 81,9\rangle$ and period of length 2 starting with branch $\mathcal{B}(4)$. Both trees have branches of bounded depth 1 , so their virtual periodicity is in fact a strict periodicity. The $\Gamma_{s}$, resp. $\Phi_{s}$, denote isoclinism families [29] [30].

However, the coclass tree of $\mathcal{G}(p, 1)$ with $p \geq 5$ has unbounded depth and contains non-metabelian groups, and the coclass tree of $\mathcal{G}(p, 1)$ with $p \geq 7$ has even unbounded width, that is the number of descendants of a fixed order increases indefinitely with growing order [26].

With the aid of kernels and targets of Artin transfer homomorphisms [3], the diagrams in Figure 2 and Figure 3 can be endowed with additional information and redrawn as structured descendant trees ([6], Figure 3.1, p. 419, and Figure 3.2, p. 422). 
The concrete examples $\mathcal{G}(2,1)$ and $\mathcal{G}(3,1)$ provide an opportunity to give a parametrized polycyclic power-commutator presentation ([31], pp. 82-84) for the complete coclass tree, mentioned in $\S 2$ as a benefit of the descendant tree concept and as a consequence of the periodicity of the pruned coclass tree. In both cases, the group $G$ is generated by two elements $x, y$ but the presentation contains the series of higher commutators $s_{j}, \quad 2 \leq j \leq n-1=\mathrm{cl}(G)$, starting with the main commutator $s_{2}=y, x$. The nilpotency is formally expressed by $s_{n}=1$, when the group is of order $|G|=p^{n}$.

For $p=2$, there are two parameters $0 \leq w, z \leq 1$ and the pc-presentation is given by

$$
\left.G^{n}(z, w)=\left\langle x, y, s_{2}, \cdots, s_{n-1}\right| x^{2}=s_{n-1}^{w}, y^{2}=s_{2}^{-1} s_{n-1}^{z},\left[s_{2}, y\right]=1, s_{2}=[y, x], s_{j}=\left[s_{j-1}, x\right] \text { for } 3 \leq j \leq n-1\right\rangle
$$

The 2-groups of maximal class, that is of coclass 1 , form three periodic infinite sequences:

- the dihedral groups, $D\left(2^{n}\right)=G^{n}(0,0), n \geq 3$, forming the mainline (with infinitely capable vertices);

- the generalized quaternion groups, $Q\left(2^{n}\right)=G^{n}(0,1), n \geq 3$, which are all terminal vertices;

- the semidihedral groups, $S\left(2^{n}\right)=G^{n}(1,0), n \geq 4$, which are also leaves.

For $p=3$, there are three parameters $0 \leq a \leq 1$ and $-1 \leq w, z \leq 1$ and the pc-presentation is given by

$G_{a}^{n}(z, w)=\left\langle x, y, s_{2}, \cdots, s_{n-1}\right| x^{3}=s_{n-1}^{w}, y^{3}=s_{2}^{-3} s_{3}^{-1} s_{n-1}^{z},\left[y, s_{2}\right]=s_{n-1}^{a}, s_{2}=[y, x], s_{j}=\left[s_{j-1}, x\right]$ for $\left.3 \leq j \leq n-1\right\rangle(14$

3 -groups with parameter $a=0$ possess an abelian maximal subgroup, those with parameter $a=1$ do not. More precisely, an existing abelian maximal subgroup is unique, except for the two extra special groups $G_{0}^{3}(0,0)$ and $G_{0}^{3}(0,1)$, where all four maximal subgroups are abelian.

In contrast to any bigger coclass $r \geq 2$, the coclass graph $\mathcal{G}(p, 1)$ exclusively contains $p$-groups $G$ with abelianization $G / G^{\prime}$ of type $(p, p)$, except for its unique isolated vertex. The case $p=2$ is distinguished by the truth of the reverse statement: Any 2 -group with abelianization of type $(2,2)$ is of coclass 1 (Taussky's Theorem ([32], p. 83).

Figure 4 shows the interface between finite 3-groups of coclass 1 and 2 of type $(3,3)$.

\subsection{Coclass 2}

The genesis of the coclass graph $\mathcal{G}(p, r)$ with $r \geq 2$ is not uniform. $p$-groups with several distinct abelianizations contribute to its constitution. For coclass $r=2$, there are essential contributions from groups $G$ with abelianizations $G / G^{\prime}$ of the types $(p, p),\left(p^{2}, p\right),(p, p, p)$, and an isolated contribution by the cyclic group of order $p^{3}$ :

$$
\mathcal{G}(p, 2)=\mathcal{G}_{(p, p)}(p, 2) \dot{\cup} \mathcal{G}_{\left(p^{2}, p\right)}(p, 2) \dot{\cup} \mathcal{G}_{(p, p, p)}(p, 2) \dot{\cup} \mathcal{G}_{\left(p^{3}\right)}(p, 2)
$$

\subsubsection{Abelianization of Type $(p, p)$}

As opposed to $p$-groups of coclass 2 with abelianization of type $\left(p^{2}, p\right)$ or $(p, p, p)$, which arise as regular descendants of abelian $p$-groups of the same types, $p$-groups of coclass 2 with abelianization of type $(p, p)$ arise from irregular descendants of a non-abelian $p$-group with coclass 1 and nuclear rank 2 .

For the prime $p=2$, such groups do not exist at all, since the dihedral group $\langle 8,3\rangle$ is coclass-settled, which is the deeper reason for Taussky's Theorem. This remarkable fact has been observed by Bagnera ([33], Part 2, 4, p. 182) in 1898 already.

For odd primes $p \geq 3$, the existence of $p$-groups of coclass 2 with abelianization of type $(p, p)$ is due to the fact that the extra special group $G_{0}^{3}(0,0)$ is not coclass-settled. Its nuclear rank equals 2 , which gives rise to a bifurcation of the descendant tree $\mathcal{T}\left(G_{0}^{3}(0,0)\right)$ into two coclass graphs. The regular component $\mathcal{T}^{1}\left(G_{0}^{3}(0,0)\right)$ is a subtree of the unique tree $\mathcal{T}^{1}\left(C_{p} \times C_{p}\right)$ in the coclass graph $\mathcal{G}(p, 1)$. The irregular component $\mathcal{T}^{2}\left(G_{0}^{3}(0,0)\right)$ becomes a subgraph $\mathcal{G}=\mathcal{G}_{(p, p)}(p, 2)$ of the coclass graph $\mathcal{G}(p, 2)$ when the connecting edges of depth 2 of the irregular immediate descendants of $G_{0}^{3}(0,0)$ are removed.

For $p=3$, this subgraph $\mathcal{G}$ is drawn in Figure 4. It has seven top level vertices of three important kinds, all having order $243=3^{5}$, which have been discovered by Bagnera ([33], Part 2, 4, pp. 182-183). 

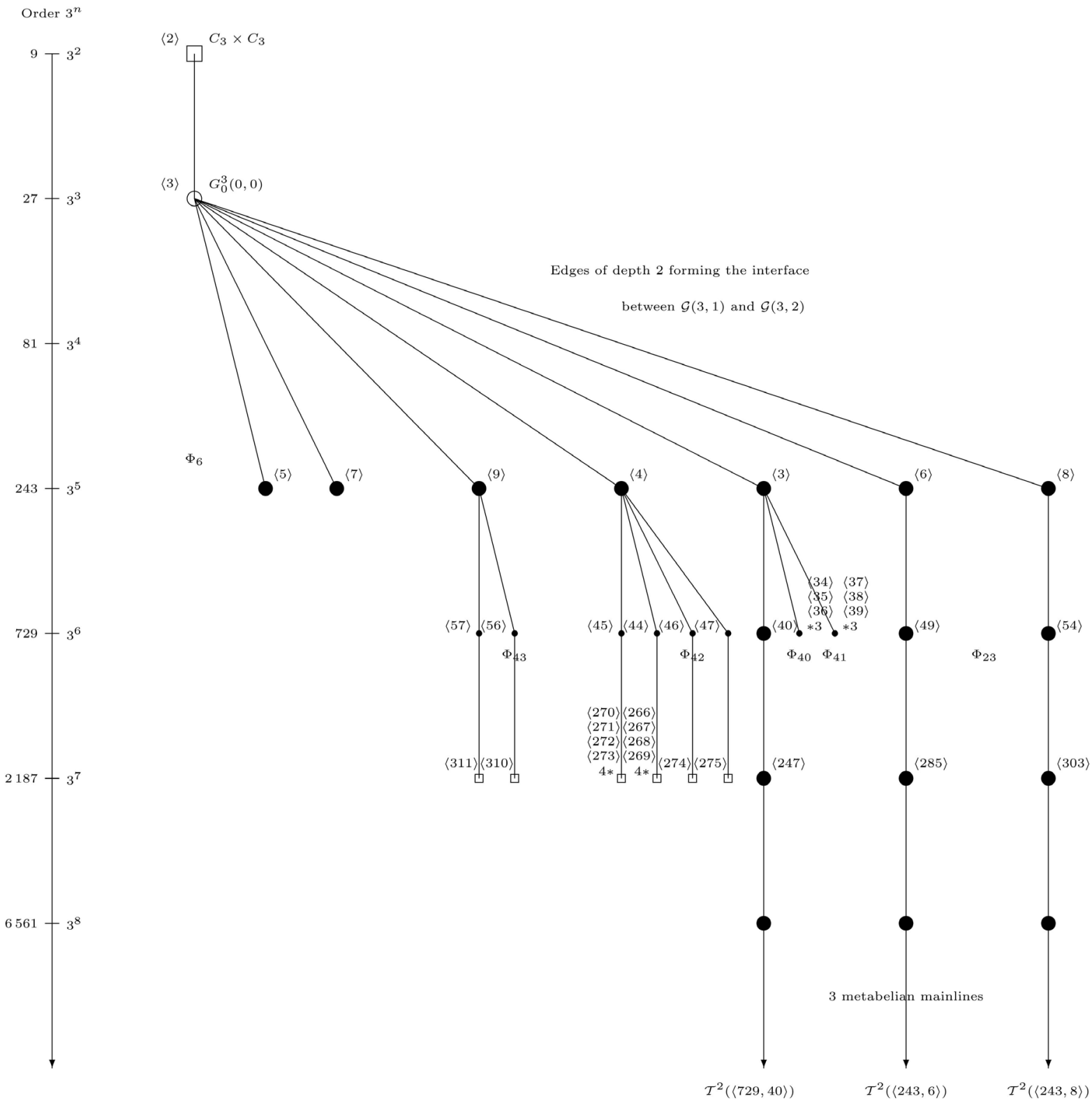

Figure 4. 3-groups of coclass 2 with abelianization $(3,3)$.

- Firstly, there are two terminal Schur $\sigma$-groups [34] $\langle 243,5\rangle$ and $\langle 243,7\rangle$ in the sporadic part $\mathcal{G}_{0}(3,2)$ of the coclass graph $\mathcal{G}(3,2)$;

- Secondly, the two groups $G=\langle 243,4\rangle$ and $G=\langle 243,9\rangle$ are roots of finite trees $\mathcal{T}^{2}(G)$ in the sporadic part $\mathcal{G}_{0}(3,2)$ (however, since they are not coclass-settled, the complete trees $\mathcal{T}(G)$ are infinite);

- And, finally, the three groups $\langle 243,3\rangle,\langle 243,6\rangle$ and $\langle 243,8\rangle$ give rise to (infinite) coclass trees, e.g., $\mathcal{T}^{2}(\langle 729,40\rangle), \mathcal{T}^{2}(\langle 243,6\rangle), \mathcal{T}^{2}(\langle 243,8\rangle)$, each having a metabelian mainline, in the coclass graph $\mathcal{G}(3,2)$. None of these three groups is coclass-settled. See $\S 21$.

Displaying additional information on kernels and targets of Artin transfers [3], we can draw these trees as structured descendant trees ([6], Figure 3.5, p. 439, Figure 3.6, p. 442, and Figure 3.7, p. 443).

Definition 10.1 Generally, a Schur group (called a closed group by I. Schur, who coined the concept) is a pro- $p$ group $G$ whose relation rank $r(G)=\operatorname{dim}_{\mathbb{F}_{p}}\left(H^{2}\left(G, \mathbb{F}_{p}\right)\right)$ coincides with its generator rank 
$d(G)=\operatorname{dim}_{\mathbb{F}_{p}}\left(H^{1}\left(G, \mathbb{F}_{p}\right)\right)$. A $\sigma$-group is a pro-p group $G$ which possesses an automorphism $\sigma \in \operatorname{Aut}(G)$ inducing the inversion $x \mapsto x^{-1}$ on its abelianization $G / G^{\prime}$. A Schur $\sigma$-group [7] [34]-[36] is a Schur group $G$ which is also a $\sigma$-group and has a finite abelianization $G / G^{\prime}$.

It should be pointed out that $\langle 243,3\rangle$ is not root of a coclass tree, since its immediate descendant $\langle 729,40\rangle=B$, which is root of a coclass tree with metabelian mainline vertices, has two siblings $\langle 729,35\rangle=I$, resp. $\langle 729,34\rangle=H$, which give rise to a single, resp. three, coclass tree(s) with non-metabelian mainline vertices having cyclic centres of order 3 and branches of considerable complexity but nevertheless of bounded depth 5 .

\subsubsection{Pro-3 Groups of Coclass 2 with Non-Trivial Centre}

Eick, Leedham-Green, Newman and O'Brien ([24], 4, Thm.4.1) have constructed a family of infinite pro-3 groups with coclass 2 having a non-trivial centre of order 3 . The members are characterized by three parameters $(f, g, h)$. Their finite quotients generate all mainline vertices with bicyclic centres of type $(3,3)$ of six coclass trees in the coclass graph $\mathcal{G}(3,2)$. The association of parameters to the roots of these six trees is given in Table 1, the tree diagrams are indicated in Figure 4 and Figure 5, and the parametrized pro-3 presentation is given by

$$
G(f, g, h)=\left\langle a, t, z \mid a^{3}=z^{f},\left[t, t^{a}\right]=z^{g}, t^{1+a+a^{2}}=z^{h}, z^{3}=1,[z, a]=1,[z, t]=1\right\rangle
$$

Figure 5 shows some finite 3-groups with coclass 2 and type $(9,3)$.

\subsubsection{Abelianization of Type $\left(p^{2}, p\right)$}

For $p=3$, the top levels of the subtree $\mathcal{T}^{2}(\langle 27,2\rangle)$ of the coclass graph $\mathcal{G}(3,2)$ are drawn in Figure 5 . The most important vertices of this tree are the eight siblings sharing the common parent $\langle 81,3\rangle$, which are of three important kinds.

- Firstly, there are three leaves $\langle 243,20\rangle,\langle 243,19\rangle,\langle 243,16\rangle$ having cyclic centre of order 9, and a single leaf $\langle 243,18\rangle$ with bicyclic centre of type $(3,3)$;

- Secondly, the group $G=\langle 243,14\rangle$ is root of a finite tree $\mathcal{T}(G)=\mathcal{T}^{2}(G)$;

- And, finally, the three groups $\langle 243,13\rangle,\langle 243,15\rangle$ and $\langle 243,17\rangle$ give rise to infinite coclass trees, e. g., $\mathcal{T}^{2}(\langle 2187,319\rangle), \quad \mathcal{T}^{2}(\langle 243,15\rangle), \mathcal{T}^{2}(\langle 243,17\rangle)$, each having a metabelian mainline, the first with cyclic centres of order 3 , the second and third with bicyclic centres of type $(3,3)$.

Here, it should be emphasized that $\langle 243,13\rangle$ is not root of a coclass tree, since aside from its descendant $\langle 2187,319\rangle$, which is root of a coclass tree with metabelian mainline vertices, it possesses five further descendants which give rise to coclass trees with non-metabelian mainline vertices having cyclic centres of order 3 and branches of considerable complexity, here partially even with unbounded depth ([24], Thm.4.2(a-b)).

\subsubsection{Abelianization of Type $(p, p, p)$}

For $p=2$, resp. $p=3$, there exists a unique coclass tree with $p$-groups of type $(p, p, p)$ in the coclass graph $\mathcal{G}(p, 2)$. Its root is the elementary abelian $p$-group of type $(p, p, p)$, that is, $\langle 8,5\rangle$, resp. $\langle 27,5\rangle$.

Table 1. Quotients of the groups $G=G(f, g, h)$.

\begin{tabular}{ccccc}
\hline Parameters & Abelianization & Class-2 quotient & Class-3 quotient & Class-4 quotient \\
\hline$(f, g, h)$ & $G / G^{\prime}$ & $G / \gamma_{3}(G)$ & $G / \gamma_{4}(G)$ & $G / \gamma_{5}(G)$ \\
\hline$(0,1,0)$ & $(3,3)$ & $\langle 27,3\rangle$ & $\langle 243,3\rangle$ & $\langle 729,40\rangle$ \\
$(0,1,2)$ & $(3,3)$ & $\langle 27,3\rangle$ & $\langle 243,6\rangle$ & $\langle 729,49\rangle$ \\
$(1,1,2)$ & $(3,3)$ & $\langle 27,3\rangle$ & $\langle 243,8\rangle$ & $\langle 729,54\rangle$ \\
$(1,0,0)$ & $(9,3)$ & $\langle 81,3\rangle$ & $\langle 243,15\rangle$ & $\langle 729,79\rangle$ \\
$(0,0,1)$ & $(9,3)$ & $\langle 81,3\rangle$ & $\langle 243,17\rangle$ & $\langle 729,84\rangle$ \\
$(0,0,0)$ & $(3,3,3)$ & $\langle 81,12\rangle$ & $\langle 243,53\rangle$ & $\langle 729,395\rangle$ \\
\hline
\end{tabular}




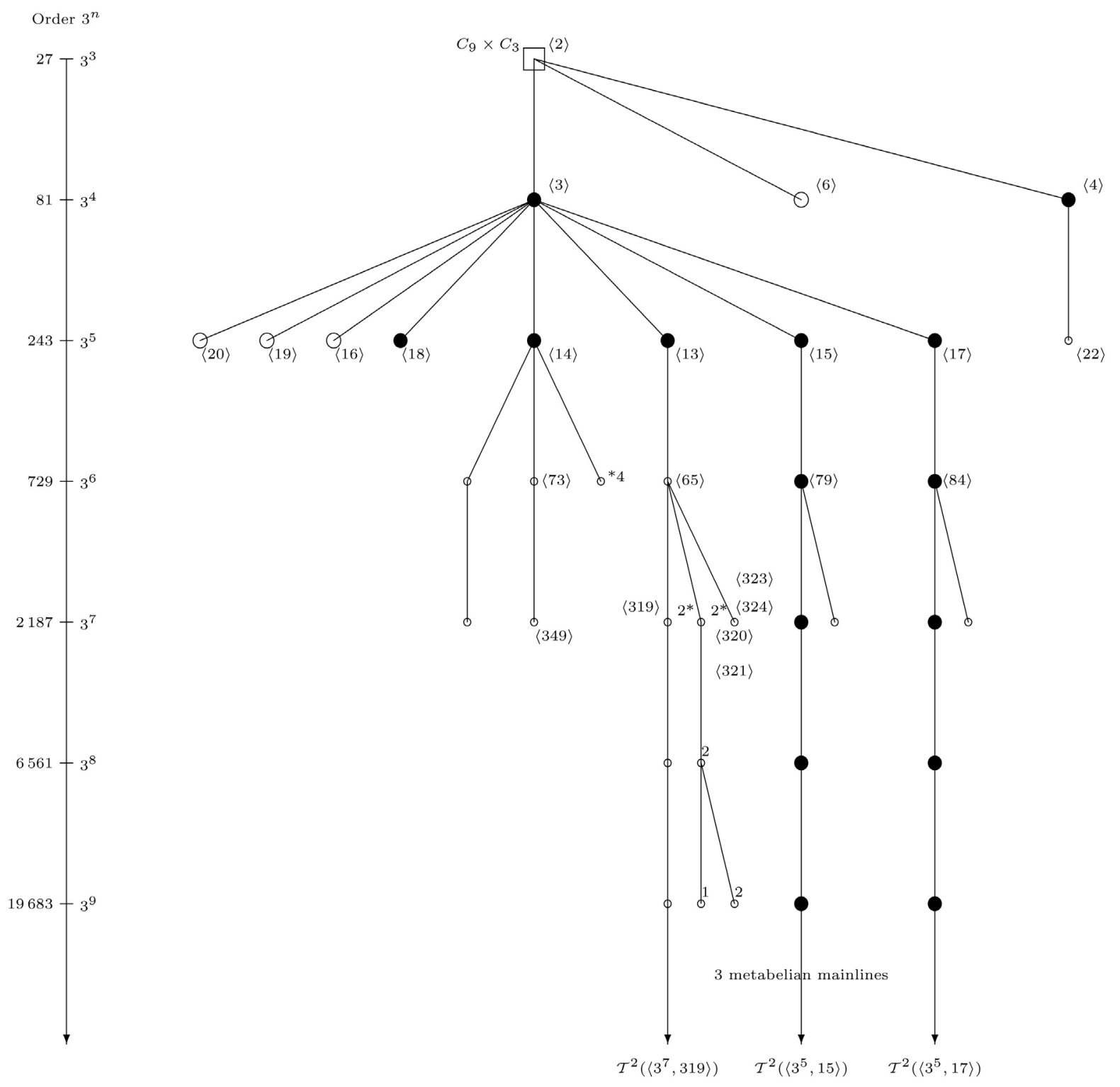

Figure 5. 3-groups of coclass 2 with abelianization $(9,3)$.

This unique tree corresponds to the pro-2 group of the family \#59 by Newman and O’Brien ([25], Appendix A, no. 59, p. 153, Appendix B, Tbl. 59, p. 165), resp. the pro-3 group given by the parameters $(f, g, h)=(0,0,0)$ in Table 1. For $p=2$, the tree is indicated in Figure 6.

Figure 6 shows some finite 2-groups of coclass 2,3,4 and type (2,2,2).

\subsection{Coclass 3}

Here again, $p$-groups with several distinct abelianizations contribute to the constitution of the coclass graph $\mathcal{G}(p, 3)$. There are regular, resp. irregular, essential contributions from groups $G$ with abelianizations $G / G^{\prime}$ of the types $\left(p^{3}, p\right),\left(p^{2}, p^{2}\right),\left(p^{2}, p, p\right),(p, p, p, p)$, resp. $(p, p),\left(p^{2}, p\right),(p, p, p)$, and an isolated contribution by the cyclic group of order $p^{4}$.

10.4.1. Abelianization of Type $(p, p, p)$

Since the elementary abelian $p$-group $C_{p} \times C_{p} \times C_{p}$ of rank 3, that is, $\langle 8,5\rangle$, resp. $\langle 27,5\rangle$, for $p=2$, resp. 


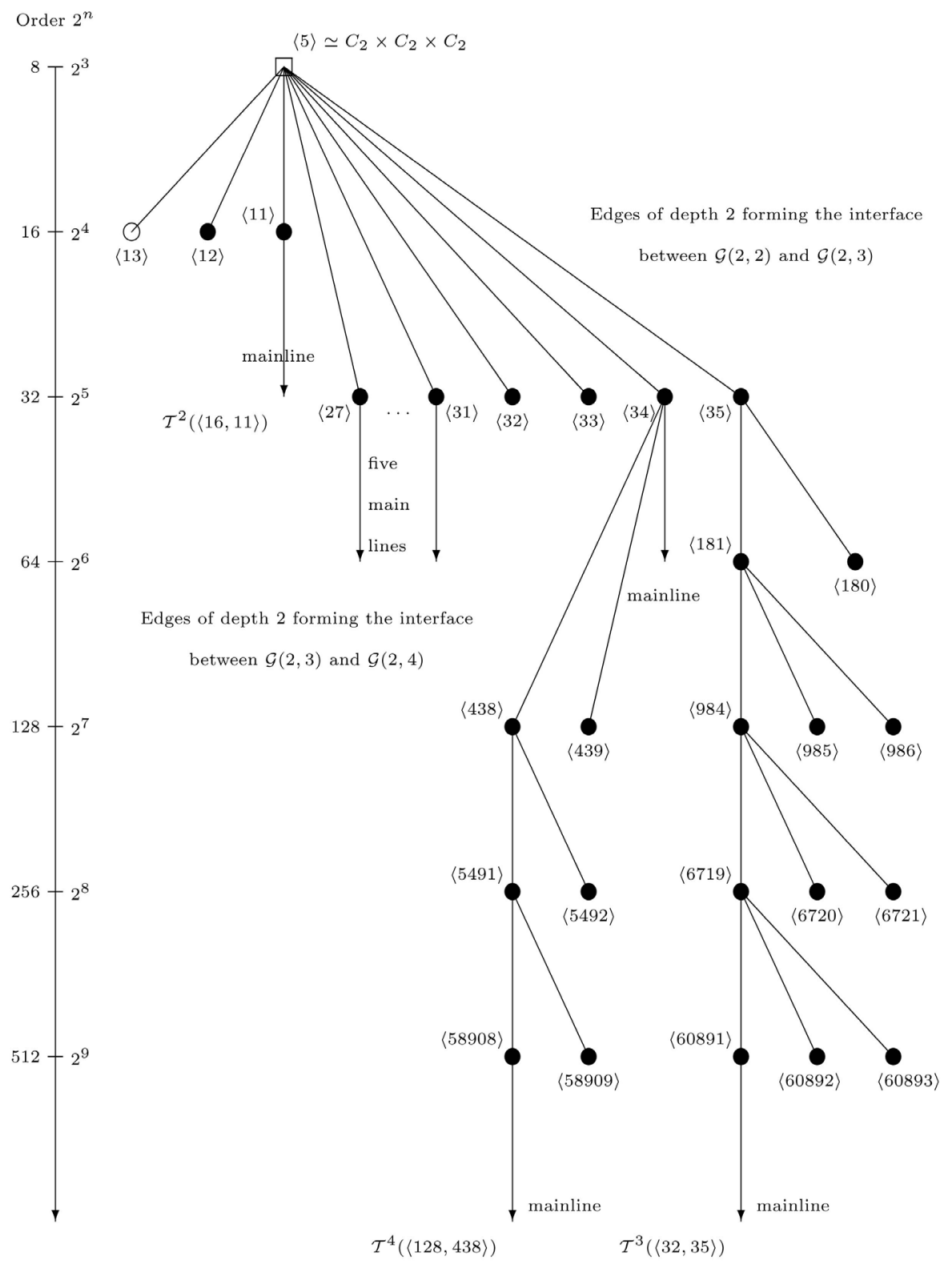

Figure 6. 2-groups of coclass 3 with abelianization $(2,2,2)$.

$p=3$, is not coclass-settled, it gives rise to a multifurcation. The regular component $\mathcal{T}^{2}\left(C_{p} \times C_{p} \times C_{p}\right)$ has been described in the section about coclass 2. The irregular component $\mathcal{T}^{3}\left(C_{p} \times C_{p} \times C_{p}\right)$ becomes a subgraph $\mathcal{G}=\mathcal{G}_{(p, p, p)}(p, 3)$ of the coclass graph $\mathcal{G}(p, 3)$ when the connecting edges of depth 2 of the irregular immediate descendants of $C_{p} \times C_{p} \times C_{p}$ are removed.

For $p=2$, this subgraph $\mathcal{G}$ is contained in Figure 6. It has nine top level vertices of order $32=2^{5}$ which can be divided into terminal and capable vertices:

- the groups $\langle 32,32\rangle$ and $\langle 32,33\rangle$ are leaves;

- the five groups $\langle 32,27 . .31\rangle$ and the two groups $\langle 32,34 . .35\rangle$ are infinitely capable.

The trees arising from the capable vertices are associated with infinite pro-2 groups by Newman and O'Brien ([25], Appendix A, no. 73-79, pp. 154-155, and Appendix B, Tbl. 73-79, pp. 167-168) in the following manner:

$\langle 32,28\rangle$ gives rise to 
$\mathcal{T}^{3}(\langle 64,140\rangle)$ associated with family \#73, and $\mathcal{T}^{3}(\langle 64,147\rangle)$ associated with family \#74;

$\mathcal{T}^{3}(\langle 32,29\rangle)$ is associated with family $\# 75$;

$\mathcal{T}^{3}(\langle 32,30\rangle)$ is associated with family $\# 76$;

$\mathcal{T}^{3}(\langle 32,31\rangle)$ is associated with family $\# 77$;

$\langle 32,34\rangle$ gives rise to

$\mathcal{T}^{3}(\langle 64,147\rangle)$ associated with family $\# 78$ (see $\S 21$ ), and finally

$\mathcal{T}^{3}(\langle 32,35\rangle)$ is associated with family \#79 (see Figure 6).

The roots of the coclass trees $\mathcal{T}^{4}(\langle 128,438\rangle)$ in Figure 6 and $\mathcal{T}^{4}(\langle 128,444 \mid 445\rangle)$ in Figure 7 are siblings.

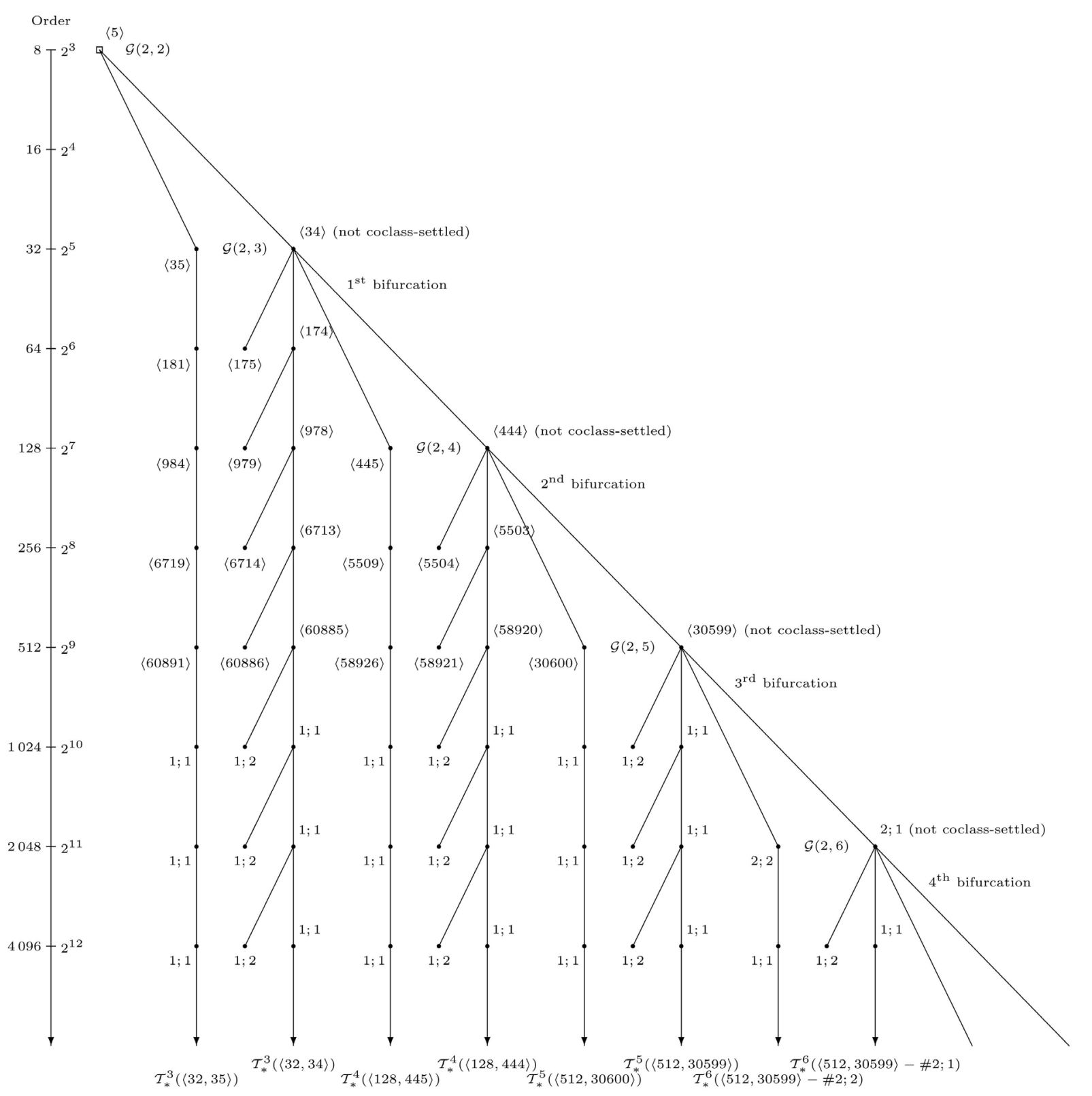

Figure 7. Periodic Bifurcations in $\mathcal{T}_{*}(\langle 32,34\rangle)$. 


\subsubsection{Hall-Senior Classification}

Seven of these nine top level vertices have been investigated by Benjamin, Lemmermeyer and Snyder ([37], 2, Tbl. 1) with respect to their occurrence as class-2 quotients $Q=G / \gamma_{3}(G)$ of bigger metabelian 2-groups $G$ of type $(2,2,2)$ and with coclass 3 , which are exactly the members of the descendant trees of the seven vertices. These authors use the classification of 2-groups by Hall and Senior [29] which is put in correspondence with the SmallGroups Library [16] [17] in Table 2. The complexity of the descendant trees of these seven vertices increases with the 2-ranks and 4-ranks indicated in Table 2, where the maximal subgroups of index 2 in $G$ are denoted by $H_{i}$, for $1 \leq i \leq 7$.

\section{History of Descendant Trees}

Descendant trees with central quotients as parents (P1) are implicit in Hall's 1940 paper [30] about isoclinism of groups. Trees with last non-trivial lower central quotients as parents (P2) were first presented by LeedhamGreen at the International Congress of Mathematicians in Vancouver, 1974 [20]. The first extensive tree diagrams have been drawn manually by Ascione, Havas and Leedham-Green (1977) [14], by Ascione (1979) [18] and by Nebelung (1989) [15]. In the former two cases, the parent definition by means of the lower exponent- $p$ central series (P3) was adopted in view of computational advantages, in the latter case, where theoretical aspects were focused, the parents were taken with respect to the usual lower central series (P2).

The kernels and targets of Artin transfer homomorphisms have recently turned out to be compatible with parent-descendant relations between finite $p$-groups and can favourably be used to endow descendant trees with additional structure [6].

\section{The Construction: $p$-Group Generation Algorithm}

The $p$-group generation algorithm by Newman [8] and O'Brien [9] [10] is a recursive process for constructing the descendant tree of an assigned finite $p$-group which is taken as the root of the tree. It is discussed in some detail in $\S \S 13-19$.

\section{Lower Exponent- $p$ Central Series}

For a finite $p$-group $G$, the lower exponent- $p$ central series (briefly lower $p$-central series) of $G$ is a descending series $\left(P_{j}(G)\right)_{j \geq 0}$ of characteristic subgroups of $G$, defined recursively by

$$
P_{0}(G):=G \text { and } P_{j}(G):=\left[P_{j-1}(G), G\right] \cdot P_{j-1}(G)^{p}, \text { for } j \geq 1
$$

Since any non-trivial finite $p$-group $G>1$ is nilpotent, there exists an integer $c \geq 1$ such that $P_{c-1}(G)>P_{c}(G)=1$ and $\mathrm{cl}_{p}(G):=c$ is called the exponent- $p$ class (briefly $p$-class) of $G$. Only the trivial

\begin{tabular}{|c|c|c|c|c|c|}
\hline $\begin{array}{l}\text { SmallGroups } \\
\text { identifier of } Q\end{array}$ & $\begin{array}{c}\text { Hall Senior } \\
\text { classification of } Q\end{array}$ & $\begin{array}{l}\text { Schur multiplier } \\
\qquad \mathcal{M}(Q)\end{array}$ & $\begin{array}{c}\text { 2-rank of } G^{\prime} \\
r_{2}\left(G^{\prime}\right)\end{array}$ & $\begin{array}{c}\text { 4-rank of } G^{\prime} \\
r_{4}\left(G^{\prime}\right)\end{array}$ & $\begin{array}{c}\text { Maximum of } \\
r_{2}\left(H_{i} / H_{i}^{\prime}\right)\end{array}$ \\
\hline$\langle 32,32\rangle$ & 32.040 & (2) & 2 & 0 & 2 \\
\hline$\langle 32,33\rangle$ & 32.041 & (2) & 2 & 0 & 2 \\
\hline$\langle 32,29\rangle$ & 32.037 & $(2,2)$ & 2 & 1 & 3 \\
\hline$\langle 32,30\rangle$ & 32.038 & $(2,2)$ & 2 & 1 & 3 \\
\hline$\langle 32,35\rangle$ & 32.035 & $(2,2)$ & 2 & 1 & 3 \\
\hline$\langle 32,28\rangle$ & 32.036 & $(2,2,2)$ & 2 & 2 & 3 \\
\hline$\langle 32,27\rangle$ & 32.033 & $(2,2,2,2)$ & 3 & 2 or 3 & 4 \\
\hline
\end{tabular}


group 1 has $\operatorname{cl}_{p}(1)=0$. Generally, for any finite $p$-group $G$, its $p$-class can be defined as $\mathrm{cl}_{p}(G):=\min \left\{c \geq 0 \mid P_{c}(G)=1\right\}$.

The complete lower $p$-central series of $G$ is therefore given by

$$
G=P_{0}(G)>\Phi(G)=P_{1}(G)>P_{2}(G)>\cdots>P_{c-1}(G)>P_{c}(G)=1
$$

since $P_{1}(G)=\left[P_{0}(G), G\right] \cdot P_{0}(G)^{p}=[G, G] \cdot G^{p}=\Phi(G)$ is the Frattini subgroup of $G$.

For the convenience of the reader and for pointing out the shifted numeration, we recall that the (usual) lower central series of $G$ is also a descending series $\left(\gamma_{j}(G)\right)_{j \geq 1}$ of characteristic subgroups of $G$, defined recursively by

$$
\gamma_{1}(G):=G \text { and } \gamma_{j}(G):=\left[\gamma_{j-1}(G), G\right], \text { for } j \geq 2
$$

As above, for any non-trivial finite $p$-group $G>1$, there exists an integer $c \geq 1$ such that $\gamma_{c}(G)>\gamma_{c+1}(G)=1$ and $\operatorname{cl}(G):=c$ is called the nilpotency class of $G$, whereas $c+1$ is called the index of nilpotency of $G$. Only the trivial group 1 has $\operatorname{cl}(1)=0$.

Thus, the complete lower central series of $G$ is given by

$$
G=\gamma_{1}(G)>G^{\prime}=\gamma_{2}(G)>\gamma_{3}(G)>\cdots>\gamma_{c}(G)>\gamma_{c+1}(G)=1
$$

since $\gamma_{2}(G)=\left[\gamma_{1}(G), G\right]=[G, G]=G^{\prime}$ is the commutator subgroup or derived subgroup of $G$.

The following rules should be remembered for the exponent- $p$ class:

Let $G$ be a finite $p$-group.

$[(\mathrm{R})]$

1) $\operatorname{cl}(G) \leq \operatorname{cl}_{p}(G)$, since the $\gamma_{j}(G)$ descend more quickly than the $P_{j}(G)$;

2) $\vartheta \in \operatorname{Hom}(G, \tilde{G})$, for some group $\tilde{G} \Rightarrow \vartheta\left(P_{j}(G)\right)=P_{j}(\vartheta(G))$, for any $j \geq 0$;

3) For any $c \geq 0$, the conditions $N \unlhd G$ and $\operatorname{cl}_{p}(G / N)=c$ imply $P_{c}(G) \leq N$;

4) For any $c \geq 0, \mathrm{cl}_{p}(G)=c \Rightarrow \mathrm{cl}_{p}\left(G / P_{k}(G)\right)=\min (k, c)$, for all $k \geq 0$, in particular, $\mathrm{cl}_{p}\left(G / P_{k}(G)\right)=k$, for all $0 \leq k \leq c$.

We point out that every non-trivial finite $G$-group $G>1$ defines a maximal path with respect to the parent definition (P3), consisting of $c$ edges,

$$
\begin{aligned}
G & =G / 1=G / P_{c}(G) \rightarrow \pi(G)=G / P_{c-1}(G) \rightarrow \pi^{2}(G)=G / P_{c-2}(G) \rightarrow \cdots \rightarrow \pi^{c-1}(G) \\
& =G / P_{1}(G) \rightarrow \pi^{c}(G)=G / P_{0}(G)=G / G=1,
\end{aligned}
$$

and ending in the trivial group $\pi^{c}(G)=1$. The last but one quotient of the maximal path of $G$ is the elementary abelian $p$-group $\pi^{c-1}(G)=G / P_{1}(G) \simeq C_{p}^{d}$ of rank $d=d(G)$, where

$d(G)=\operatorname{dim}_{\mathbb{F}_{p}}\left(H^{1}\left(G, \mathbb{F}_{p}\right)\right)$ denotes the generator rank of $G$.

\section{4. $p$-Covering Group, $p$-Multiplicator and Nucleus}

Let $G$ be a finite $p$-group with $d$ generators. Our goal is to compile a complete list of pairwise nonisomorphic immediate descendants of $G$. It turned out that all immediate descendants can be obtained as quotients of a certain extension $G^{*}$ of $G$ which is called the $p$-covering group of $G$ and can be constructed in the following manner.

We can certainly find a presentation of $G$ in the form of an exact sequence

$$
1 \rightarrow R \rightarrow F \rightarrow G \rightarrow 1
$$

where $F$ denotes the free group with $d$ generators and $\vartheta: F \rightarrow G$ is an epimorphism with kernel $R:=\operatorname{ker}(\vartheta)$. Then $R \triangleleft F$ is a normal subgroup of $F$ consisting of the defining relations for $G \simeq F / R$. For 
elements $r \in R$ and $f \in F$, the conjugate $f^{-1} r f \in R$ and thus also the commutator $[r, f]=r^{-1} f^{-1} r f \in R$ are contained in $R$. Consequently, $R^{*}:=[R, F] \cdot R^{p}$ is a characteristic subgroup of $R$, and the $p$-multiplicator $R / R^{*}$ of $G$ is an elementary abelian $p$-group, since

$$
[R, R] \cdot R^{p} \leq[R, F] \cdot R^{p}=R^{*} \unlhd R
$$

Now we can define the $p$-covering group of $G$ by

$$
G^{*}:=F / R^{*}
$$

and the exact sequence

$$
1 \rightarrow R / R^{*} \rightarrow F / R^{*} \rightarrow F / R \rightarrow 1
$$

shows that $G^{*}$ is an extension of $G$ by the elementary abelian $p$-multiplicator. We call

$$
\mu(G):=\operatorname{dim}_{\mathbb{F}_{p}}\left(R / R^{*}\right)
$$

the $p$-multiplicator rank of $G$.

Let us assume now that the assigned finite $p$-group $G=F / R$ is of $p$-class $\operatorname{cl}_{p}(G)=c$. Then the conditions $R \unlhd F$ and $\mathrm{cl}_{p}(F / R)=c$ imply $P_{c}(F) \leq R$, according to the rule (R3), and we can define the nucleus of $G$ by

$$
P_{c}\left(G^{*}\right)=P_{c}(F) \cdot R^{*} / R^{*} \leq R / R^{*}
$$

as a subgroup of the $p$-multiplicator. Consequently, the nuclear rank

$$
v(G):=\operatorname{dim}_{\mathbb{F}_{p}}\left(P_{c}\left(G^{*}\right)\right) \leq \mu(G)
$$

of $G$ is bounded from above by the $p$-multiplicator rank.

\section{Allowable Subgroups of the $p$-Multiplicator}

As before, let $G$ be a finite $p$-group with $d$ generators. Any $p$-elementary abelian central extension $1 \rightarrow Z \rightarrow H \rightarrow G \rightarrow 1$ of $G$ by a $p$-elementary abelian subgroup $Z \leq \zeta_{1}(H)$ such that $d(H)=d(G)=d$ is a quotient of the $p$-covering group $G^{*}$ of $G$.

The reason is that there exists an epimorphism $\psi: F \rightarrow H$ such that $\vartheta=\omega \circ \psi$, where $\omega: H \rightarrow G=H / Z$ denotes the canonical projection. Consequently, we have $R=\operatorname{ker}(\vartheta)=\operatorname{ker}(\omega \circ \psi)=\psi^{-1}(Z)$ and thus $\psi(R)=\psi\left(\psi^{-1}(Z)\right)=Z$. Further, $\psi\left(R^{p}\right) \leq Z^{p}=1$, since $Z$ is $p$-elementary, and $\psi(R, F) \leq[Z, Z]=1$, since $Z$ is central. Together this shows that $\psi\left(R^{*}\right)=\psi\left([R, F] \cdot R^{p}\right)=1$ and thus $\psi$ induces the desired epimorphism $\psi^{*}: G^{*} \rightarrow H$ such that $H \simeq G^{*} / \operatorname{ker}\left(\psi^{*}\right)$.

In particular, an immediate descendant $H$ of $G$ is a $p$-elementary abelian central extension

$$
1 \rightarrow P_{c-1}(H) \rightarrow H \rightarrow G \rightarrow 1
$$

of $G$, since

$$
1=P_{c}(H)=\left[P_{c-1}(H), H\right] \cdot P_{c-1}(H)^{p} \text { implies } P_{c-1}(H)^{p}=1 \text { and } P_{c-1}(H) \leq \zeta_{1}(H)
$$

where $c=\mathrm{cl}_{p}(H)$.

A subgroup $M / R^{*} \leq R / R^{*}$ of the $p$-multiplicator of $G$ is called allowable if it is given by the kernel $M / R^{*}=\operatorname{ker}\left(\psi^{*}\right)$ of an epimorphism $\psi^{*}: G^{*} \rightarrow H$ onto an immediate descendant $H$ of $G$. An equivalent characterization is that $1<M / R^{*}<R / R^{*}$ is a proper subgroup which supplements the nucleus 


$$
\left(M / R^{*}\right) \cdot\left(P_{c}(F) \cdot R^{*} / R^{*}\right)=R / R^{*}
$$

Therefore, the first part of our goal to compile a list of all immediate descendants of $G$ is done, when we have constructed all allowable subgroups of $R / R^{*}$ which supplement the nucleus $P_{c}\left(G^{*}\right)=P_{c}(F) \cdot R^{*} / R^{*}$, where $c=\mathrm{cl}_{p}(G)$. However, in general the list

$$
\left\{F / M \mid M / R^{*} \leq R / R^{*} \text { is allowable }\right\}
$$

where $G^{*} /\left(M / R^{*}\right)=\left(F / R^{*}\right) /\left(M / R^{*}\right) \simeq F / M$ will be redundant, due to isomorphisms $F / M_{1} \simeq F / M_{2}$ among the immediate descendants.

\section{Orbits under Extended Automorphisms}

Two allowable subgroups $M_{1} / R^{*}$ and $M_{2} / R^{*}$ are called equivalent if the quotients $F / M_{1} \simeq F / M_{2}$, that are the corresponding immediate descendants of $G$, are isomorphic.

Such an isomorphism $\varphi: F / M_{1} \simeq F / M_{2}$ between immediate descendants of $G=F / R$ with $c=\operatorname{cl}_{p}(G)$ has the property that

$$
\varphi\left(R / M_{1}\right)=\varphi\left(P_{c}\left(F / M_{1}\right)\right)=P_{c}\left(\varphi\left(F / M_{1}\right)\right)=P_{c}\left(F / M_{2}\right)=R / M_{2}
$$

and thus induces an automorphism $\alpha \in \operatorname{Aut}(G)$ of $G$ which can be extended to an automorphism $\alpha^{*} \in \operatorname{Aut}\left(G^{*}\right)$ of the $p$-covering group $G^{*}=F / R^{*}$ of $G$. The restriction of this extended automorphism $\alpha^{*}$ to the $p$-multiplicator $R / R^{*}$ of $G$ is determined uniquely by $\alpha$. Since

$$
\alpha^{*}\left(M / R^{*}\right) \cdot P_{c}\left(F / R^{*}\right)=\alpha^{*}\left[M / R^{*} \cdot P_{c}\left(F / R^{*}\right)\right]=\alpha^{*}\left(R / R^{*}\right)=R / R^{*}
$$

according to the rule (R2), each extended automorphism $\alpha^{*} \in \operatorname{Aut}\left(G^{*}\right)$ induces a permutation $\tilde{\alpha}$ of the allowable subgroups $M / R^{*} \leq R / R^{*}$. We define

$$
P:=\langle\tilde{\alpha} \mid \alpha \in \operatorname{Aut}(G)\rangle
$$

to be the permutation group generated by all permutations induced by automorphisms of $G$. Then the map $\operatorname{Aut}(G) \rightarrow P, \alpha \mapsto \tilde{\alpha}$ is an epimorphism and the equivalence classes of allowable subgroups $M / R^{*} \leq R / R^{*}$ are precisely the orbits of allowable subgroups under the action of the permutation group $P$.

Eventually, our goal to compile a list $\left\{F / M_{i} \mid 1 \leq i \leq N\right\}$ of all immediate descendants of $G$ will be done, when we select a representative $M_{i} / R^{*}$ for each of the $N$ orbits of allowable subgroups of $R / R^{*}$ under the action of $P$. This is precisely what the $p$-group generation algorithm does in a single step of the recursive procedure for constructing the descendant tree of an assigned root.

\section{Capable $p$-Groups and Step Sizes}

We recall from $\S 6$ that a finite $p$-group $G$ is called capable (or extendable) if it possesses at least one immediate descendant, otherwise it is called terminal (or a leaf). As mentioned in $\S 8$ already, the nuclear rank $v(G)$ of $G$ admits a decision about the capability of $G$ :

- $G$ is terminal if and only if $v(G)=0$;

- $G$ is capable if and only if $v(G) \geq 1$.

In the case of capability, $G=F / R$ has immediate descendants of $v=v(G)$ different step sizes $1 \leq s \leq v$, in dependence on the index

$$
\left(R / R^{*}: M / R^{*}\right)=p^{s}
$$

of the corresponding allowable subgroup $M / R^{*}$ in the $p$-multiplicator $R / R^{*}$. When $G$ is of order $|G|=p^{n}$, then an immediate descendant of step size $s$ is of order 


$$
\#(F / M)=\left(F / R^{*}: M / R^{*}\right)=\left(F / R^{*}: R / R^{*}\right) \cdot\left(R / R^{*}: M / R^{*}\right)=|G| \cdot p^{s}=p^{n} \cdot p^{s}=p^{n+s}
$$

For the related phenomenon of multifurcation of a descendant tree at a vertex $G$ with nuclear rank $v(G) \geq 2$ see $\S 8$ on multifurcation and coclass graphs.

The $p$-group generation algorithm provides the flexibility to restrict the construction of immediate descendants to those of a single fixed step size $1 \leq s \leq v$, which is very convenient in the case of huge descendant numbers (see the next section).

\section{Numbers of Immediate Descendants}

We denote the number of all immediate descendants, resp. immediate descendants of step size $s$, of $G$ by $N$, resp. $N_{s}$. Then we have $N=\sum_{s=1}^{v} N_{s}$. As concrete examples, we present some interesting finite metabelian $p$-groups with extensive sets of immediate descendants, using the SmallGroups identifiers and additionally pointing out the numbers $0 \leq C_{s} \leq N_{s}$ of capable immediate descendants in the usual format

$$
\left(N_{1} / C_{1} ; \cdots ; N_{v} / C_{v}\right)
$$

as given by actual implementations of the $p$-group generation algorithm in the computational algebra systems GAP and MAGMA. These invariants completely determine the local structure of the descendant tree $\mathcal{T}(G)$.

First, let $p=2$. We begin with groups having abelianization of type $(2,2,2)$. See Figure 6 .

- The group $\langle 32,35\rangle$ of coclass 3 has ranks $v=1, \mu=5$ and descendant numbers $(4 / 1), N=4$. See $\S$ 21 ;

- The group $\langle 32,34\rangle$ of coclass 3 has ranks $v=2, \mu=6$ and descendant numbers $(6 / 1 ; 19 / 6), N=25$. See $\S 21$;

- The group $\langle 32,27\rangle$ of coclass 3 has ranks $v=3, \mu=7$ and descendant numbers $(12 / 2 ; 70 / 25 ; 104 / 85), \quad N=186$;

Next, let $p=3$. We consider groups having abelianization of type $(3,3)$. See Figure 4;

- The group $\langle 27,3\rangle$ of coclass 1 has ranks $v=2, \mu=4$ and descendant numbers $(4 / 1 ; 7 / 5), N=11$;

- The group $\langle 243,3\rangle=\langle 27,3\rangle-\# 2 ; 1$ of coclass 2 has ranks $v=2, \mu=4$ and descendant numbers $(10 / 6 ; 15 / 15), \quad N=25$;

- One of its immediate descendants, the group $B=\langle 729,40\rangle=\langle 243,3\rangle-\# 1 ; 7$, has ranks $v=2, \mu=5$ and descendant numbers $(16 / 2 ; 27 / 4), \quad N=43$.

In contrast, groups with abelianization of type $(3,3,3)$ are partially located beyond the limit of actual computability.

- The group $\langle 81,12\rangle$ of coclass 2 has ranks $v=2, \mu=7$ and descendant numbers $(10 / 2 ; 100 / 50)$, $N=110$;

- The group $\langle 243,37\rangle$ of coclass 3 has ranks $v=5, \mu=9$ and descendant numbers $(35 / 3 ; 2783 / 186 ; 81711 / 10202 ; 350652 / 202266 ; \cdots), N>4 \times 10^{5}$ unknown;

- The group $\langle 729,122\rangle$ of coclass 4 has ranks $v=8, \mu=11$ and descendant numbers $(45 / 3 ; 117919 / 1377 ; \cdots), \quad N>10^{5}$ unknown.

\section{Schur Multiplier}

Via the isomorphism

$$
\mathbb{Q} / \mathbb{Z} \rightarrow \mu_{\infty}, \quad \frac{n}{d} \mapsto \exp \left(\frac{n}{d} \cdot 2 \pi i\right)
$$

group

$$
\mathbb{Q} / \mathbb{Z}=\left\{\frac{n}{d} \cdot \mathbb{Z} \mid d \geq 1,0 \leq n \leq d-1\right\}
$$


can be viewed as the additive analogue of the multiplicative group

$$
\mu_{\infty}=\left\{z \in \mathbb{C} \mid z^{d}=1 \text { for some integer } d \geq 1\right\}
$$

of all roots of unity.

Let $p$ be a prime number and $G$ be a finite $p$-group with presentation $G=F / R$ as in the previous section. Then the second cohomology group

$$
M(G):=H^{2}(G, \mathbb{Q} / \mathbb{Z})
$$

of the $G$-module $\mathbb{Q} / \mathbb{Z}$ is called the Schur multiplier of $G$. It can also be interpreted as the quotient group

$$
M(G)=(R \cap[F, F]) /[F, R]
$$

Shafarevich ([38], 6, p. 146) has proved that the difference between the relation rank

$r(G)=\operatorname{dim}_{\mathbb{F}_{p}}\left(H^{2}\left(G, \mathbb{F}_{p}\right)\right)$ of $G$ and the generator rank $d(G)=\operatorname{dim}_{\mathbb{F}_{p}}\left(H^{1}\left(G, \mathbb{F}_{p}\right)\right)$ of $G$ is given by the minimal number of generators of the Schur multiplier of $G$, that is

$$
r(G)-d(G)=d(M(G))
$$

Boston and Nover ([39], 3.2, Prop. 2) have shown that

$$
\mu\left(G_{j}\right)-v\left(G_{j}\right) \leq r(G)
$$

for all quotients $G_{j}:=G / P_{j}(G)$ of $p$-class $\operatorname{cl}_{p}\left(G_{j}\right)=j, j \geq 0$, of a pro- $p$ group $G$ with finite abelianization $G / G^{\prime}$.

Furthermore, Blackhurst (in the appendix On the nucleus of certain $p$-groups of a paper by Boston, Bush and Hajir [35]) has proved that a non-cyclic finite $p$-group $G$ with trivial Schur multiplier $M(G)$ is a terminal vertex in the descendant tree $\mathcal{T}(1)$ of the trivial group 1, that is,

$$
M(G)=1 \Rightarrow v(G)=0
$$

We conclude this section by giving two examples.

- A finite $p$-group $G$ has a balanced presentation $r(G)=d(G)$ if and only if $r(G)-d(G)=0=d(M(G))$, that is, if and only if its Schur multiplier $M(G)=1$ is trivial. Such a group is called a Schur group [7] [34]-[36] and it must be a leaf in the descendant tree $\mathcal{T}(1)$;

- A finite $p$-group $G$ satisfies $r(G)=d(G)+1$ if and only if $r(G)-d(G)=1=d(M(G))$, that is, if and only if it has a non-trivial cyclic Schur multiplier $M(G)$. Such a group is called a Schur +1 group.

\section{Pruning Strategies}

For searching a particular group in a descendant tree $\mathcal{T}(R)$ by looking for patterns defined by the kernels and targets of Artin transfer homomorphisms [6], it is frequently adequate to reduce the number of vertices in the branches of a dense tree with high complexity by sifting groups with desired special properties, for example

$[(\mathrm{F})]$

1) filtering the $\sigma$-groups (see Definition 10.1);

2) eliminating a set of certain transfer kernel types (TKTs, see ([6], pp. 403-404));

3) cancelling all non-metabelian groups (thus restricting to the metabelian skeleton);

4) removing metabelian groups with cyclic centre (usually of higher complexity);

5) cutting off vertices whose distance from the mainline (depth) exceeds some lower bound;

6) combining several different sifting criteria.

The result of such a sieving procedure is called a pruned descendant tree $\mathcal{T}_{*}(R)<\mathcal{T}(R)$ with respect to the desired set of properties.

However, in any case, it should be avoided that the mainline of a coclass tree is eliminated, since the result would be a disconnected infinite set of finite graphs instead of a tree. We expand this idea further in the 
following detailed discussion of new phenomena.

\section{Striking News: Periodic Bifurcations in Trees}

We begin this section about brand-new discoveries with the most recent example of periodic bifurcations in trees of 2-groups. It has been found on the 17th of January 2015, motivated by a search for metabelian 2-class tower groups [40] of complex quadratic fields [41] and complex bicyclic biquadratic Dirichlet fields [42].

\subsection{Finite 2-Groups $G$ with $G / G^{\prime} \simeq(2,2,2)$}

The 2-groups under investigation are three-generator groups with elementary abelian commutator factor group of type $(2,2,2)$. As shown in Figure 6 of $\S 10$, all such groups are descendants of the abelian root $\langle 8,5\rangle$. Among its immediate descendants of step size 2, there are three groups which reveal multifurcation. $\langle 32,27\rangle$ has nuclear rank $v=3$, giving rise to 3 -fold multifurcation. The two groups $\langle 32,28\rangle$ and $\langle 32,34\rangle$ possess the required nuclear rank $v=2$ for bifurcation. Due to the arithmetical origin of the problem, we focused on the latter, $G:=\langle 32,34\rangle$, and constructed an extensive finite part of its pruned descendant tree $\mathcal{T}_{*}(G)$, using the $p$-group generation algorithm [8]-[10] as implemented in the computational algebra system Magma [13] [43] [44]. All groups turned out to be metabelian.

Remark 21.1 Since our primary intention is to provide a sound group theoretic background for several phenomena discovered in class field theory and algebraic number theory, we eliminated superfluous brushwood in the descendant trees to avoid unnecessary complexity.

The selected sifting process for reducing the entire descendant tree $\mathcal{T}(G)$ to the pruned descendant tree $\mathcal{T}_{*}(G)$ filters all vertices which satisfy one of the conditions in Equation (44) or (49), and essentially consists of pruning strategy (F2), more precisely, of

1) omitting all the 14 terminal step size- 2 descendants, and 5, resp. 4, of the 6 capable step size- 2 descendants, together with their complete descendant trees, in Theorem 21.1, resp. Corollary 21.1, and

2) eliminating all, resp. 4, of the 5 terminal step size-1 descendants in Theorem 21.1, resp. Corollary 21.1.

Denote by $x, y, z$ the generators of a finite 2-group $G=\langle x, y, z\rangle$ with abelian type invariants $(2,2,2)$. We fix an ordering of the seven maximal normal subgroups by putting

$$
\begin{aligned}
& S_{1}=\left\langle y, z, G^{\prime}\right\rangle, S_{2}=\left\langle z, x, G^{\prime}\right\rangle, S_{3}=\left\langle x, y, G^{\prime}\right\rangle, S_{4}=\left\langle x, y z, G^{\prime}\right\rangle, S_{5}=\left\langle y, z x, G^{\prime}\right\rangle, \\
& S_{6}=\left\langle z, x y, G^{\prime}\right\rangle, S_{7}=\left\langle x y, y z, G^{\prime}\right\rangle .
\end{aligned}
$$

Just within this subsection, we select a special designation for a TKT [[6], p. 403-404] whose first layer consists exactly of all these seven planes in the 3-dimensional $\mathbb{F}_{2}$-vector space $G / G^{\prime}$, in any ordering.

Definition 21.1 The transfer kernel type (TKT) $\varkappa=\left[\varkappa_{0} ; \varkappa_{1} ; \varkappa_{2} ; \varkappa_{3}\right]$ is called a permutation if all seven members of the first layer $\varkappa_{1}$ are maximal subgroups of $G$ and there exists a permutation $\sigma \in S_{7}$ such that $\varkappa_{1}=\left(S_{\sigma(j)}\right)_{1 \leq j \leq 7}$.

For brevity, we give 2-logarithms of abelian type invariants in the following theorem and we denote iteration by formal exponents, for instance, $1^{3}:=(1,1,1) \hat{=}(2,2,2),\left(1^{3}\right)^{4}:=\left(1^{3}, 1^{3}, 1^{3}, 1^{3}\right), 0^{7}:=(0,0,0,0,0,0,0)$ and $(j+2, j+1) \hat{=}\left(2^{j+2}, 2^{j+1}\right)$. Further, we eliminate an initial anomaly of generalized identifiers by putting $G-\# 2 ; 1:=G-\# 2 ; 8$ and $G-\# 2 ; 2:=G-\# 2 ; 9$, formally.

Theorem 21.1 Let $\ell$ be a positive integer bounded from above by 10 .

1) In the descendant tree $\mathcal{T}(G)$ of $G=\langle 32,34\rangle$, there exists a unique path of length $\ell$,

$$
G=\delta^{0}(G) \leftarrow \delta^{1}(G) \leftarrow \cdots \leftarrow \delta^{\ell}(G)
$$

of (reverse) directed edges with uniform step size 2 such that $\delta^{j}(G)=\pi\left(\delta^{j+1}(G)\right)$, for all $0 \leq j \leq \ell-1$ (along the path, $\delta$ is a section of the surjection $\pi$ ), and all the vertices

$$
\delta^{j}(G)=G(-\# 2 ; 1)^{j}
$$

of this path share the following common invariants: 
- the transfer kernel type with layer $\varkappa_{1}$ containing three 2-cycles (and nearly a permutation, except for the first component which is total, $0 \hat{=} \delta^{j}(G)$ ),

$$
\varkappa\left(\delta^{j}(G)\right)=\left[1 ;\left(0, S_{6}, S_{5}, S_{7}, S_{3}, S_{2}, S_{4}\right) ; 0^{7} ; 0\right]
$$

- the 2-multiplicator rank and the nuclear rank, giving rise to the bifurcation,

$$
\mu\left(\delta^{j}(G)\right)=6, \quad v\left(\delta^{j}(G)\right)=2
$$

- and the counters of immediate descendants,

$$
N_{1}\left(\delta^{j}(G)\right)=6, \quad C_{1}\left(\delta^{j}(G)\right)=1, \quad N_{2}\left(\delta^{j}(G)\right)=19, \quad C_{2}\left(\delta^{j}(G)\right)=6
$$

determining the local structure of the descendant tree.

2) A few other invariants of the vertices $\delta^{j}(G)$ depend on the superscript $j$,

- the 2-logarithm of the order, the nilpotency class and the coclass,

$$
\log _{2}\left(\operatorname{ord}\left(\delta^{j}(G)\right)\right)=2 j+5, \quad \operatorname{cl}\left(\delta^{j}(G)\right)=j+2, \quad \operatorname{cc}\left(\delta^{j}(G)\right)=j+3
$$

- a single component of layer $\tau_{1}$, three components of layer $\tau_{2}$, and layer $\tau_{3}$ of the transfer target type

$$
\tau\left(\delta^{j}(G)\right)=\left[1^{3} ;\left((j+2, j+2),\left(1^{3}\right)^{6}\right) ;\left((j+2, j+1)^{3},\left(1^{3}\right)^{4}\right) ;(j+1, j+1)\right]
$$

In view of forthcoming number theoretic applications, we add the following

Corollary 21.1 Let $0 \leq j \leq \ell$ be a non-negative integer.

1) The regular component $\mathcal{T}^{j+3}\left(\delta^{j}(G)\right)$ of the descendant tree $\mathcal{T}\left(\delta^{j}(G)\right)$ is a coclass tree which contains a unique periodic sequence whose vertices $V_{j, k}:=\delta^{j}(G)(-\# 1 ; 1)^{k}-\# 1 ; 2$ with $k \geq 0$ are characterized by a permutation TKT

$$
\mathcal{T}\left(V_{j, k}\right)=\left[1 ;\left(S_{1}, S_{6}, S_{5}, S_{7}, S_{3}, S_{2}, S_{4}\right) ; 0^{7} ; 0\right]
$$

with a single fixed point $S_{1}$ and the same three 2-cycles $\left(S_{2}, S_{6}\right),\left(S_{3}, S_{5}\right),\left(S_{4}, S_{7}\right)$ as in the mainline TKT of Equation (44).

2) The irregular component $\mathcal{T}^{j+4}\left(\delta^{j}(G)\right)$ of the descendant tree $\mathcal{T}\left(\delta^{j}(G)\right)$ is a forest which contains a unique second coclass tree $\mathcal{T}^{j+4}\left(\delta^{j}(G)-\# 2 ; 2\right)$ whose mainline vertices $M_{j+1, k}:=\delta^{j}(G)-\# 2 ; 2(-\# 1 ; 1)^{k}$ with $k \geq 0$ possess the same permutation TKT as in Equation (49), apart from the first coclass tree $\mathcal{T}^{j+4}\left(\delta^{j}(G)-\# 2 ; 1\right)$, where $\delta^{j}(G)-\# 2 ; 1=\delta^{j+1}(G)$, whose mainline vertices $\delta^{j+1}(G)(-\# 1 ; 1)^{k}$ with $k \geq 0$ share the TKT in Equation (44).

Proof. (of Theorem 21.1, Corollary 21.1 and Theorem 21.2)

The $p$-group generation algorithm [8]-[10] as implemented in the Magma computational algebra system [13] [43] [44] was employed to construct the pruned descendant tree $\mathcal{T}_{*}(G)$ with root $G=\langle 32,34\rangle$ which we defined as the disjoint union of all pruned coclass trees $\mathcal{T}_{*}^{j+3}\left(\delta^{j}(G)\right)$ with the successive descendants $\delta^{j}(G)=G(-\# 2 ; 1)^{j}, 0 \leq j \leq 10$, of step size 2 of $G$ as roots. Using the well-known virtual periodicity [1] [2] of each coclass tree $\mathcal{T}^{j+3}\left(\delta^{j}(G)\right)$, which turned out to be strict and of the smallest possible length 1 , the vertical construction was terminated at nilpotency class 12 , considerably deeper than the point where periodicity sets in. The horizontal construction was extended up to coclass 13, where the amount of CPU time started to become annoying.

Within the frame of our computations, the periodicity was not restriced to bifurcations only: It seems that the pruned (or maybe even the entire) descendant trees $\mathcal{T}_{*}\left(\delta^{j}(G)\right)$ are all isomorphic to $\mathcal{T}_{*}(G)$ as graphs. This is visualized impressively by Figure 7.

The extent to which we constructed the pruned descendant tree suggests the following conjecture.

Conjecture 21.1 Theorem 21.1, Corollary 21.1 and Theorem 21.2 remain true for an arbitrarily large positive 
integer $\ell$, not necessarily bounded by 10 .

Remark 21.2 We must emphasize that the root $\langle 8,5\rangle$ in Figure 7 is drawn for the sake of completeness only, and that the mainline of the coclass tree $\mathcal{T}^{3}(\langle 32,35\rangle)$ is exceptional, since

- its root is not a descendant of $G$ and

- the TKT of its vertices $M_{0, k}:=\langle 32,35\rangle(-\# 1 ; 1)^{k}$ with $k \geq 0$,

$$
\varkappa\left(M_{0, k}\right)=\left[1 ;\left(S_{1}, S_{2}, S_{5}, S_{4}, S_{3}, S_{6}, S_{7}\right) ; 0^{7} ; 0\right]
$$

is a permutation with 5 fixed points and only a single 2 -cycle $\left(S_{3}, S_{5}\right)$.

One-parameter polycyclic pc-presentations for all occurring groups are given as follows.

1) For the mainline vertices of the coclass tree $\mathcal{T}^{3}(\langle 32,34\rangle)$ with class $c \geq 3$, that is, starting with $\langle 64,174\rangle$ and excluding the root $\langle 32,34\rangle$, by

$$
\begin{aligned}
\delta^{0}(G)(-\# 1 ; 1)^{c-2}=G_{3}^{c}:= & \left\langle x, y, z, s_{2}, \cdots, s_{c}, t_{2}\right| s_{2}=[y, x], t_{2}=[z, x], s_{j}=\left[s_{j-1}, x\right] \text { for } 3 \leq j \leq c, \\
& \left.x^{2}=1, y^{2}=s_{2} s_{3}, z^{2}=t_{2}, s_{j}^{2}=s_{j+1} s_{j+2} \text { for } 2 \leq j \leq c-2, s_{c-1}^{2}=s_{c}\right\rangle .
\end{aligned}
$$

2) For the mainline vertices of the coclass tree $\mathcal{T}^{4}(\langle 128,444\rangle)$ with class $c \geq 3$ by

$$
\begin{aligned}
\delta^{1}(G)(-\# 1 ; 1)^{c-3}=G_{4}^{c}:= & \left\langle x, y, z, s_{2}, \cdots, s_{c}, t_{2}, t_{3}\right| s_{2}=[y, x], t_{2}=[z, x], s_{j}=\left[s_{j-1}, x\right] \text { for } 3 \leq j \leq c, t_{3}=t_{2}, x,(52) \\
& \left.x^{2}=1, y^{2}=s_{2} s_{3}, z^{2}=t_{2} t_{3}, s_{j}^{2}=s_{j+1} s_{j+2} \text { for } 2 \leq j \leq c-2, s_{c-1}^{2}=s_{c}, t_{2}^{2}=t_{3}\right\rangle .
\end{aligned}
$$

3) For the mainline vertices of the coclass tree $\mathcal{T}^{5}(\langle 512,30599\rangle)$ with class $c \geq 4$ by

$$
\begin{aligned}
\delta^{2}(G)(-\# 1 ; 1)^{c-4}=G_{5}^{c}:= & \left\langle x, y, z, s_{2}, \cdots, s_{c}, t_{2}, t_{3}, t_{4}\right| s_{2}=[y, x], t_{2}=[z, x], s_{j}=\left[s_{j-1}, x\right] \text { for } 3 \leq j \leq c, \\
& t_{3}=\left[t_{2}, x\right], t_{4}=\left[t_{3}, x\right], x^{2}=1, y^{2}=s_{2} s_{3}, z^{2}=t_{2} t_{3}, s_{j}^{2}=s_{j+1} s_{j+2} \text { for } 2 \leq j \leq c-2, \\
& \left.s_{c-1}^{2}=s_{c}, t_{2}^{2}=t_{3} t_{4}, t_{3}^{2}=t_{4}\right\rangle .
\end{aligned}
$$

Theorem 21.2 For higher coclass $4 \leq r \leq \ell+3$ the presentations (52) and (53) can be generalized in the shape of a two-parameter polycyclic pc-presentation for class $r-1 \leq c \leq \ell+2$.

$$
\begin{aligned}
\delta^{r-3}(G)(-\# 1 ; 1)^{c-r+1}=G_{r}^{c}:= & \left\langle x, y, z, s_{2}, \cdots, s_{c}, t_{2}, \cdots, t_{r-1}\right| s_{2}=[y, x], t_{2}=[z, x], s_{j}=\left[s_{j-1}, x\right] \text { for } 3 \leq j \leq c, \\
& t_{k}=\left[t_{k-1}, x\right] \text { for } 3 \leq k \leq r-1, x^{2}=1, y^{2}=s_{2} s_{3}, z^{2}=t_{2} t_{3}, s_{j}^{2}=s_{j+1} s_{j+2} \\
& \text { for } \left.2 \leq j \leq c-2, s_{c-1}^{2}=s_{c}, t_{k}^{2}=t_{k+1} t_{k+2} \text { for } 2 \leq k \leq r-3, t_{r-2}^{2}=t_{r-1}\right\rangle .
\end{aligned}
$$

To obtain a presentation for the vertices $\delta^{r-3}(G)(-\# 1 ; 1)^{c-r}-\# 1 ; 2, c \geq r$, at depth 1 in the distinguished periodic sequence whose vertices are characterized by the permutation TKT (49), we must only add the single relation $x^{2}=s_{c}$ to the presentation (54) of the mainline vertices of the coclass tree $\mathcal{T}^{r}\left(\delta^{r-3}(G)\right)$ given in Theorem 21.2.

\subsection{Finite 3-Groups $G$ with $G / G^{\prime} \simeq(3,3)$}

We continue this section with periodic bifurcations in trees of 3-groups, which have been discovered in 2012 and 2013 [45]-[47], inspired by a search for 3-class tower groups of complex quadratic fields [7] [48] [49], which must be Schur $\sigma$-groups.

These 3-groups are two-generator groups of coclass at least 2 with elementary abelian commutator quotient of type $(3,3)$. As shown in Figure 4 of $\S 10$, all such groups are descendants of the extra special group $\langle 27,3\rangle$. Among its 7 immediate descendants of step size 2, there are only two groups which satisfy the requirements arising from the arithmetical background.

The two groups $\langle 243,6\rangle$ and $\langle 243,8\rangle$ do not show multifurcation themselves but they are not coclasssettled either, since their immediate mainline descendants $Q=\langle 729,49\rangle$ and $U=\langle 729,54\rangle$ possess the required 
nuclear rank $v=2$ for bifurcation. We constructed an extensive finite part of their pruned descendant trees $\mathcal{T}_{*}(G), G \in\{Q, U\}$, using the $p$-group generation algorithm [8]-[10] as implemented in the computational algebra system Magma [13] [43] [44].

Denote by $x, y$ the generators of a finite 3-group $G=\langle x, y\rangle$ with abelian type invariants $(3,3)$. We fix an ordering of the four maximal normal subgroups by putting

$$
H_{1}=\left\langle y, G^{\prime}\right\rangle, \quad H_{2}=\left\langle x, G^{\prime}\right\rangle, \quad H_{3}=\left\langle x y, G^{\prime}\right\rangle, \quad H_{4}=\left(x y^{2}, G^{\prime}\right)
$$

Within this subsection, we make use of special designations for transfer kernel types (TKTs) which were defined generally in [[6], p. 403-404] and more specifically for the present scenario in [4] [50].

We are interested in the unavoidable mainline vertices with TKTs c.18, $\varkappa=(0,1,2,2)$, resp. c.21, $\varkappa=(2,0,3,4)$, and, above all, in most essential vertices of depth 1 forming periodic sequences with TKTs E.6, $\varkappa=(1,1,2,2)$ and E.14, $\varkappa=(3,1,2,2) \sim(4,1,2,2)$, resp. E. $8, \varkappa=(2,2,3,4)$ and E.9, $\varkappa=(2,3,3,4) \sim(2,4,3,4)$, and we want to eliminate the numerous and annoying vertices with TKTs H.4, $\varkappa=(2,1,2,2)$, resp. G.16, $\varkappa=(2,1,3,4)$.

We point out that, for instance E.9, $\varkappa=(2,3,3,4) \sim(2,4,3,4)$, is a shortcut for the layer $\varkappa_{1}=\left(H_{2}, H_{3}, H_{3}, H_{4}\right) \sim\left(H_{2}, H_{4}, H_{3}, H_{4}\right)$ of the complete (layered) TKT $\varkappa=\left[\varkappa_{0} ; \varkappa_{1} ; \varkappa_{2}\right]$.

Remark 21.3 We choose the following sifting strategy for reducing the entire descendant tree $\mathcal{T}(G)$ to the pruned descendant tree $\mathcal{T}_{*}(G)$. We filter all vertices which, firstly, are $\sigma$-groups, and secondly satisfy one of the conditions in Equations (58) or (67), whence the process is a combination (F6) $=(\mathrm{F} 1)+(\mathrm{F} 2)+(\mathrm{F} 5)$ and consists of

1) keeping all of the 3 terminal step size- 2 descendants, which are exactly the Schur $\sigma$-groups, and omitting 2 of the 3 capable step size- 2 descendants having TKT H.4, resp. G.16, together with their complete descendant trees, and

2) eliminating 2 of the 5 terminal step size- 1 descendants having TKT c.18, resp. c.21, and 2 of the 3 capable step size-1 descendants having TKT H.4, resp. G.16, in Theorem 21.3.

For brevity, we give 3-logarithms of abelian type invariants in the following theorem and we denote iteration by formal exponents, for instance, $1^{3}:=(1,1,1) \hat{=}(3,3,3),(2,1) \hat{=}(9,3),(2,1)^{3}:=((2,1),(2,1),(2,1))$, and $(j+2, j+1) \hat{=}\left(3^{j+2}, 3^{j+1}\right)$. Further, we eliminate some initial anomalies of generalized identifiers by putting $\langle 243,8\rangle-\# 1 ; 1:=\langle 243,8\rangle-\# 1 ; 3,\langle 729,54\rangle-\# 1 ; 2|4:=\langle 729,54\rangle-\# 1 ; 4| 2$,

$\langle 729,54\rangle-\# 2 ; 2|4:=\langle 729,54\rangle-\# 2 ; 4| 2,\langle 729,54\rangle-\# 2 ; 1:=\langle 729,54\rangle-\# 2 ; 3$,

$\langle 729,49\rangle(-\# 2 ; 1-\# 1 ; 1)^{j}-\# 1 ; 1:=\langle 729,49\rangle(-\# 2 ; 1-\# 1 ; 1)^{j}-\# 1 ; 2$,

$\langle 729,49\rangle(-\# 2 ; 1-\# 1 ; 1)^{j}-\# 1 ; 4|5| 6:=\langle 729,49\rangle(-\# 2 ; 1-\# 1 ; 1)^{j}-\# 1 ; 5|6| 7$, formally.

Theorem 21.3 Let $\ell$ be a positive integer bounded from above by 8 .

1) In the descendant tree $\mathcal{T}(G)$ of $G=\langle 243,6\rangle$, resp. $G=\langle 243,8\rangle$, there exists a unique path of length $2 \ell$,

$$
G=\delta^{0}(G) \leftarrow \delta^{1}(G) \leftarrow \cdots \leftarrow \delta^{2 \ell}(G)
$$

of (reverse) directed edges of alternating step sizes 1 and 2 such that $\delta^{i}(G)=\pi\left(\delta^{i+1}(G)\right)$, for all $0 \leq i \leq 2 \ell-1$, and all the vertices with even superscript $i=2 j, j \geq 0$,

$$
\delta^{2 j}(G)=G(-\# 1 ; 1-\# 2 ; 1)^{j}
$$

resp. all the vertices with odd superscript $i=2 j+1 \quad j \geq 0$,

$$
\delta^{2 j+1}(G)=G(-\# 1 ; 1-\# 2 ; 1)^{j}-\# 1 ; 1
$$

of this path share the following common invariants, respectively:

- the uniform (w.r.t. $i$ ) transfer kernel type, containing a total component $0 \hat{=} \delta^{i}(G)$, 


$$
\varkappa\left(\delta^{i}(G)\right)=[1 ;(0,1,2,2) \operatorname{resp} .(2,0,3,4) ; 0]
$$

- the 2-multiplicator rank and the nuclear rank,

$$
\mu\left(\delta^{2 j}(G)\right)=3, \quad v\left(\delta^{2 j}(G)\right)=1
$$

resp., giving rise to the bifurcation for odd $i=2 j+1$,

$$
\mu\left(\delta^{2 j+1}(G)\right)=4, \quad v\left(\delta^{2 j+1}(G)\right)=2
$$

- and the counters of immediate descendants,

$$
N_{1}\left(\delta^{2 j}(G)\right)=4, \quad C_{1}\left(\delta^{2 j}(G)\right)=4
$$

resp.

$$
N_{1}\left(\delta^{2 j+1}(G)\right)=8, \quad C_{1}\left(\delta^{2 j+1}(G)\right)=3, \quad N_{2}\left(\delta^{2 j+1}(G)\right)=6, \quad C_{2}\left(\delta^{2 j+1}(G)\right)=3
$$

determining the local structure of the descendant tree.

2) A few other invariants of the vertices $\delta^{i}(G)$ depend on the superscript $i$,

- the 3-logarithm of the order, the nilpotency class and the coclass,

$$
\log _{3}\left(\operatorname{ord}\left(\delta^{2 j}(G)\right)\right)=3 j+5, \quad \operatorname{cl}\left(\delta^{2 j}(G)\right)=2 j+3, \quad \operatorname{cc}\left(\delta^{2 j}(G)\right)=j+2
$$

resp.

$$
\log _{3}\left(\operatorname{ord}\left(\delta^{2 j+1}(G)\right)\right)=3 j+6, \quad \operatorname{cl}\left(\delta^{2 j+1}(G)\right)=2 j+4, \quad \operatorname{cc}\left(\delta^{2 j+1}(G)\right)=j+2
$$

- a single component of layer $\tau_{1}$ and the layer $\tau_{2}$ of the transfer target type

$$
\tau\left(\delta^{2 j}(G)\right)=\left[1^{2} ;\left((j+2, j+1), 1^{3},(2,1)^{2}\right) \operatorname{resp} .\left((2,1),(j+2, j+1),(2,1)^{2}\right) ;(j+1, j+1,1)\right]
$$

resp.

$$
\tau\left(\delta^{2 j+1}(G)\right)=\left[1^{2} ;\left((j+2, j+2), 1^{3},(2,1)^{2}\right) \operatorname{resp} .\left((2,1),(j+2, j+2),(2,1)^{2}\right) ;(j+2, j+1,1)\right]
$$

Theorem 21.3 provided the scaffold of the pruned descendant tree $\mathcal{T}_{*}(G)$ of $G=\langle 243, n\rangle$, for $n \in\{6,8\}$, with mainlines and periodic bifurcations.

With respect to number theoretic applications, however, the following Corollaries 21.2 and 21.3 are of the greatest importance.

Corollary 21.2 Let $0 \leq i \leq 2 \ell$ be a non-negative integer.

Whereas the vertices with even superscript $i=2 j, j \geq 0$, that is, $\delta^{2 j}(G)=G(-\# 1 ; 1-\# 2 ; 1)^{j}$, are merely links in the distinguished path, the vertices with odd superscript $i=2 j+1, j \geq 0$, that is, $\delta^{2 j+1}(G)=G(-\# 1 ; 1-\# 2 ; 1)^{j}-\# 1 ; 1$, reveal the essential periodic bifurcations with the following properties.

1) The regular component $\mathcal{T}^{j+2}\left(\delta^{2 j+1}(G)\right)$ of the descendant tree $\mathcal{T}\left(\delta^{2 j+1}(G)\right)$ is a coclass tree which contains the mainline,

$$
M_{j, k}:=\delta^{2 j+1}(G)(-\# 1 ; 1)^{k} \text { with } k \geq 0
$$

which entirely consists of $\sigma$-groups, and three distinguished periodic sequences whose vertices

$$
V_{j, k}:=\delta^{2 j+1}(G)(-\# 1 ; 1)^{k}-\# 1 ; 2|4| 6 \text { resp. } 4|5| 6 \text { with } k \geq 0
$$

are $\sigma$-groups exactly for even $k=2 k^{\prime} \geq 0$ and are characterized by the following TKTs $\varkappa\left(V_{j, k}\right)=\left[1 ; \varkappa_{1} ; 0\right]$ with layer $\varkappa_{1}$ given by

$$
\varkappa_{1} \in\{(1,1,2,2),(3,1,2,2),(4,1,2,2)\} \text { resp. } \varkappa_{1} \in\{(2,2,3,4),(2,3,3,4),(2,4,3,4)\}
$$


which deviate from the mainline TKT of Equation (58) in a single component only.

2) The irregular component $\mathcal{T}^{j+3}\left(\delta^{2 j+1}(G)\right)$ of the descendant tree $\mathcal{T}\left(\delta^{2 j+1}(G)\right)$ is a forest which contains a bunch of 3 isolated Schur $\sigma$-groups

$$
S_{j}:=\delta^{2 j+1}(G)-\# 2 ; 2|4| 6 \text { resp. } 4|5| 6,
$$

which possess the same TKTs as in Equation (67), and additionally contains the root of the next coclass tree $\mathcal{T}^{j+3}\left(\delta^{2 j+1}(G)-\# 2 ; 1\right)$, where $\delta^{2 j+1}(G)-\# 2 ; 1=\delta^{2(j+1)}(G)$, whose mainline vertices $\delta^{2(j+1)}(G)(-\# 1 ; 1)^{k}$ with $k \geq 0$ share the TKT in Equation (58).

The metabelian 3-groups forming the three distinguished periodic sequences

$$
V_{0,2 k}=\delta^{1}(G)(-\# 1 ; 1)^{2 k}-\# 1 ; 2|4| 6 \text { resp. } 4|5| 6 \text { with } k \geq 0
$$

of the pruned coclass tree $\mathcal{T}_{*}^{2}(G)$ in Corollary 21.2, for $i=0$, belong to the few groups for which all immediate descendants with respect to the parent definition (P4) are known (we did not use this kind of descendants up to now.) Since all groups in $\mathcal{T}(G) \backslash \mathcal{T}^{2}(G)$ are of derived length 3, the set of these descendants can be defined in the following way.

Definition 21.2 Let $P$ be a finite metabelian $p$-group. Then the set of all finite non-metabelian $p$-groups $D$ whose second derived quotient $D / D^{\prime \prime}$ is isomorphic to $P$ is called the cover $\operatorname{cov}(P)$ of $P$. The subset $\operatorname{cov}_{*}(P)$ consisting of all Schur $\sigma$-groups in $\operatorname{cov}(P)$ is called the balanced cover of $P$.

Corollary 21.3 For $0 \leq k \leq \ell$, the group $V_{0,2 k}$, which does not have a balanced presentation, possesses a finite cover of cardinality \# $\operatorname{cov}\left(V_{0,2 k}\right)=k+1$ and a unique Schur $\sigma$-group in its balanced cover with $\# \operatorname{cov}_{*}\left(V_{0,2 k}\right)=1$. More precisely, the covers are given explicitly by

$$
\begin{aligned}
& \operatorname{cov}\left(V_{0,2 k}\right)=\left\{V_{j, 2(k-j)} \mid 1 \leq j \leq k\right\} \cup\left\{S_{k}\right\}, \\
& \operatorname{cov}_{*}\left(V_{0,2 k}\right)=\left\{S_{k}\right\} .
\end{aligned}
$$

The arrows in Figure 8 and Figure 9 indicate the projections $\pi$ from all members $D$ of a cover $\operatorname{cov}(P)$ onto the common metabelianization $P$, that is, in the sense of the parent definition (P4), from the descendants $D$ onto the parent $P=\pi(D)$.

Proof. (of Theorem 21.3, Corollary 21.2, Corollary 21.3 and Theorem 21.4)

The $p$-group generation algorithm [8]-[10], which is implemented in the computational algebra system Magma [13] [43] [44], was used for constructing the pruned descendant trees $\mathcal{T}_{*}(G)$ with roots $G=\langle 243,6 \mid 8\rangle$ which were defined as the disjoint union of all pruned coclass trees $\mathcal{T}_{*}^{j+2}\left(\delta^{2 j+1}(G)\right)$ of the descendants $\delta^{2 j+1}(G)=G(-\# 1 ; 1-\# 2 ; 1)^{j}-\# 1 ; 1,0 \leq j \leq 10$, of $G$ as roots, together with 4 siblings in the irregular component $\mathcal{T}_{*}^{j+3}\left(\delta^{2 j+1}(G)\right), 3$ of them Schur $\sigma$-groups with $\mu=2$ and $v=0$. Using the strict periodicity [1] [2] of each pruned coclass tree $\mathcal{T}_{*}^{j+2}\left(\delta^{2 j+1}(G)\right)$, which turned out to be of length 2, the vertical construction was terminated at nilpotency class 19 , considerably deeper than the point where periodicity sets in. The horizontal construction was extended up to coclass 10 , where the consumption of CPU time became daunting.

Within the frame of our computations, the periodicity was not restriced to bifurcations only: It seems that the pruned (or maybe even the entire) descendant trees $\mathcal{T}_{*}\left(\delta^{2 j+1}(G)\right)$ are all isomorphic to $\mathcal{T}_{*}\left(\delta^{1}(G)\right)$ as graphs. This is visualized impressively by Figure 8 and Figure 9, where the following notation (not to be confused with layers) is used

$$
\varkappa_{1}=(4,1,2,2), \quad \varkappa_{2}=(3,1,2,2), \quad \varkappa_{3}=(1,1,2,2), \quad \varkappa_{0}=(0,1,2,2)
$$

resp. 


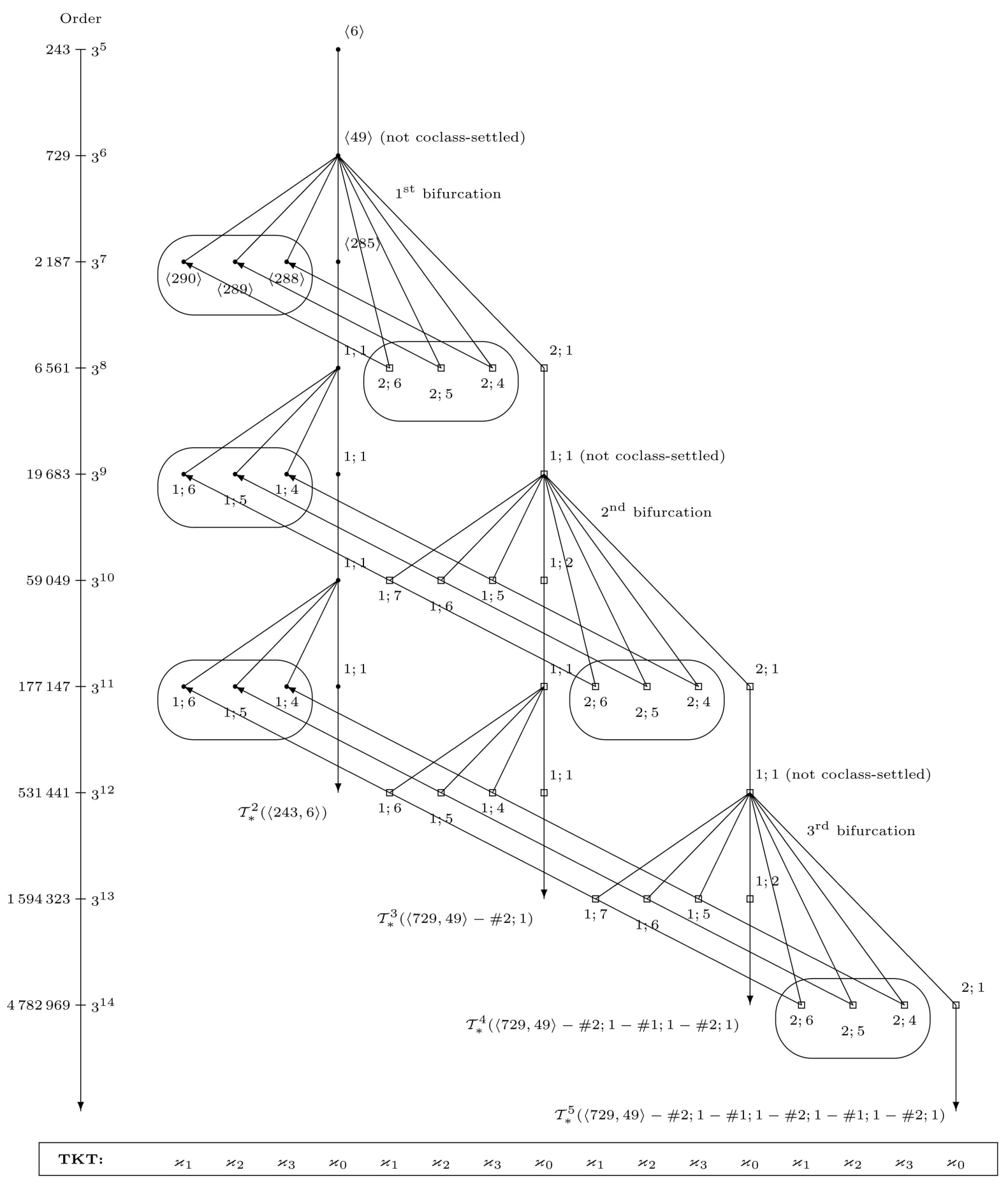

Figure 8. Periodic bifurcations in $\mathcal{T}_{*}(\langle 243,6\rangle)$.

$$
\varkappa_{1}=(2,4,3,4), \quad \varkappa_{2}=(2,3,3,4), \quad \varkappa_{3}=(2,2,3,4), \quad \varkappa_{0}=(2,0,3,4)
$$

Similarly as in the previous section, the extent to which we constructed the pruned descendant trees suggests the following conjecture.

Conjecture 21.2 Theorem 21.3, Corollary 21.2 and Corollary 21.3 remain true for an arbitrarily large positive integer $\ell$, not necessarily bounded by 8 . 


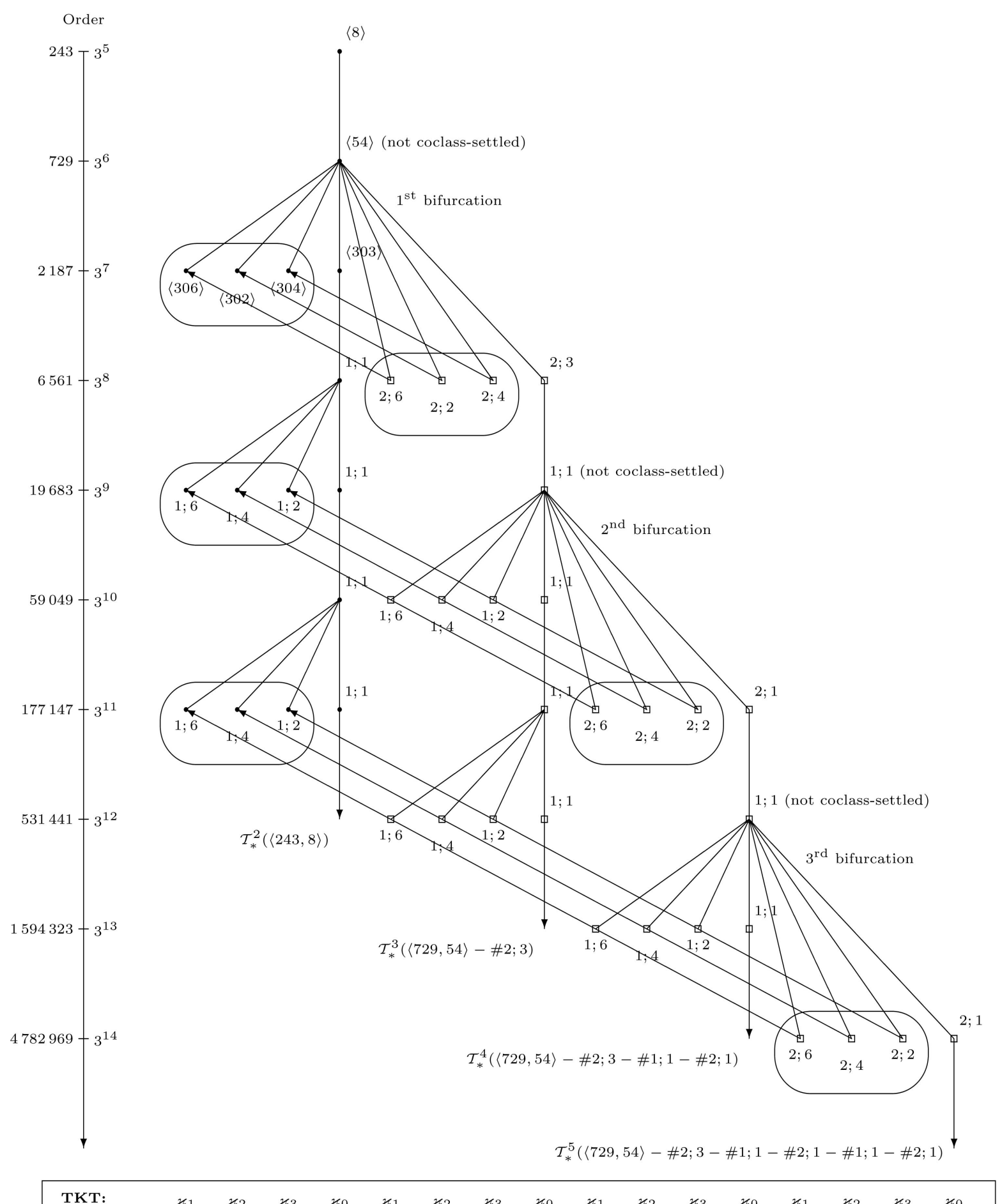

Figure 9. Periodic bifurcations in $\mathcal{T}_{*}(\langle 243,8\rangle)$.

One-parameter polycyclic pc-presentations for the groups in the first three pruned coclass trees of $\mathcal{T}_{*}(\langle 243,6\rangle)$ are given as follows.

1) For the metabelian vertices of the pruned coclass tree $\mathcal{T}_{*}^{2}\left(\delta^{0}(G)\right)$ with class $c \geq 5$, that is, starting with $\langle 2187,285\rangle$ and excluding the root $\delta^{0}(G)=\langle 243,6\rangle$ and its descendant $Q=\langle 729,49\rangle$, by 


$$
\begin{aligned}
\delta^{0}(G)(-\# 1 ; 1)^{c-3}=G_{2}^{c}(0,0), & \\
G_{2}^{c}(z, w):= & \left\langle x, y, s_{2}, \cdots, s_{c}, t_{3}\right| s_{2}=[y, x], s_{j}=\left[s_{j-1}, x\right] \text { for } 3 \leq j \leq c, t_{3}=\left[s_{2}, y\right], s_{j}^{3}=s_{j+2}^{2} s_{j+3} \\
& \left.\quad \text { for } 2 \leq j \leq c-3, s_{c-2}^{3}=s_{c}^{2}, t_{3}^{3}=1, x^{3}=s_{c}^{w}, y^{3}=s_{3}^{2} s_{4} s_{c}^{z}\right\rangle .
\end{aligned}
$$

2) For the non-metabelian vertices of the pruned coclass tree $\mathcal{T}_{*}^{3}\left(\delta^{2}(G)\right)$ with class $c \geq 5$, and including the Schur $\sigma$-groups, which are siblings of the root, by

$$
\begin{aligned}
\delta^{2}(G)(-\# 1 ; 1)^{c-5}=G_{3}^{c}(0,0), & \\
G_{3}^{c}(z, w):= & \left\langle x, y, s_{2}, \cdots, s_{c}, t_{3}, u_{5}\right| s_{2}=[y, x], s_{j}=\left[s_{j-1}, x\right] \text { for } 3 \leq j \leq c, t_{3}=\left[s_{2}, y\right], u_{5}=\left[s_{3}, y\right]=\left[s_{4}, y\right], \\
& \left.\quad\left[s_{3}, s_{2}\right]=u_{5}^{2}, t_{3}^{3}=u_{5}^{2}, s_{2}^{3}=s_{4}^{2} s_{5} u_{5}, s_{j}^{3}=s_{j+2}^{2} s_{j+3} \text { for } 3 \leq j \leq c-3, s_{c-2}^{3}=s_{c}^{2}, x^{3}=s_{c}^{w}, y^{3}=s_{3}^{2} s_{4} s_{c}^{z}\right\rangle .
\end{aligned}
$$

3) For the non-metabelian vertices of the pruned coclass tree $\mathcal{T}_{*}^{4}\left(\delta^{4}(G)\right)$ with class $c \geq 7$, and including the Schur $\sigma$-groups, which are siblings of the root, by

$$
\begin{aligned}
& \delta^{4}(G)(-\# 1 ; 1)^{c-7}=G_{4}^{c}(0,0), \\
& G_{4}^{c}(z, w):=\left\langle x, y, s_{2}, \cdots, s_{c}, t_{3}, u_{5}, u_{7}\right| s_{2}=[y, x], s_{j}=\left[s_{j-1}, x\right] \text { for } 3 \leq j \leq c, t_{3}=\left[s_{2}, y\right], \\
& \\
& u_{5}=\left[s_{4}, y\right], u_{7}=\left[s_{6}, y\right],\left[s_{3}, s_{2}\right]=u_{5}^{2} u_{7}^{2},\left[s_{3}, y\right]=u_{5} u_{7}^{2},\left[s_{5}, y\right]=u_{7}^{2}, \\
& {\left[s_{4}, s_{2}\right]=u_{7}^{2},\left[s_{5}, s_{2}\right]=u_{7}^{2},\left[s_{4}, s_{3}\right]=u_{7}, s_{2}^{3}=s_{4}^{2} s_{5} u_{5}, s_{3}^{3}=s_{5}^{2} s_{6} u_{7}^{2},} \\
& t_{3}^{3}=u_{5}^{2} u_{7}^{2}, u_{5}^{3}=u_{7}^{2}, s_{j}^{3}=s_{j+2}^{2} s_{j+3} \text { for } 4 \leq j \leq c-3, s_{c-2}^{3}=s_{c}^{2}, \\
& \left.x^{3}=s_{c}^{w}, y^{3}=s_{3}^{2} s_{4} s_{c}^{2}\right\rangle .
\end{aligned}
$$

The parameter $c$ is the nilpotency class of the group, and the parameters $0 \leq w \leq 1$ and $0 \leq z \leq 2$ determine

- the location of the group on the descendant tree, and

- the transfer kernel type (TKT) of the group, as follows:

$G_{r}^{c}(0,0)$ lies on the mainline (this is the so-called mainline principle) and has TKT c. $18, \varkappa=(0,1,2,2)$, whereas all the other groups belong to periodic sequences or are isolated Schur $\sigma$-groups:

$G_{r}^{c}(0,1)$ possesses TKT E.6, $\varkappa=(1,1,2,2)$,

$G_{r}^{c}(1,0)$ and $G_{r}^{c}(2,0)$ have TKT H.4, $\varkappa=(2,1,2,2)$, and lie outside of the pruned tree,

$G_{r}^{c}(1,1)$ and $G_{r}^{c}(2,1)$ have TKT E.14, $\varkappa=(3,1,2,2) \sim(4,1,2,2)$.

In Figure 10, resp. Figure 11, we have drawn the lattice of normal subgroups of $G_{3}^{5}(z, w)$, resp. $G_{4}^{7}(z, w)$.

The upper and lower central series, $\zeta(G), \gamma(G)$, of these groups form subgraphs whose relative position justifies the names of these series, as visualized impressively by Figure 10 and Figure 11 .

Generators $x, y \in G / G^{\prime}, s_{2}, s_{3}, t_{3}, \cdots \in G^{\prime} / G^{\prime \prime}$, and $u_{5}, u_{7} \in G^{\prime \prime}$, are carefully selected independently from individual isomorphism types and placed in locations which illustrate the structure of the groups. Furthermore, the normal lattice of the metabelianization $G / G^{\prime \prime}$ is also included as a subgraph simply by putting $u_{5}=1$.

We conclude with a theorem concerning the central series and some fundamental properties of the Schur $\sigma$ groups which we encountered among all the groups under investigation.

Theorem 21.4

Let $0 \leq j \leq l-1$ be an integer. There exist exactly 6 pairwise non-isomorphic groups $G$ of order $3^{3 j+8}$, class $2 j+5$, coclass $j+3$, having fixed derived length 3 , such that

1) the factors of their upper central series are given by

$$
\zeta_{k+1}(G) / \zeta_{k}(G) \simeq \begin{cases}(3,3), & \text { for } k=2 j+4, \\ (3), & \text { for } 1 \leq k \leq 2 j+3, \\ \left(3,3^{j+2}\right), & \text { for } k=0,\end{cases}
$$




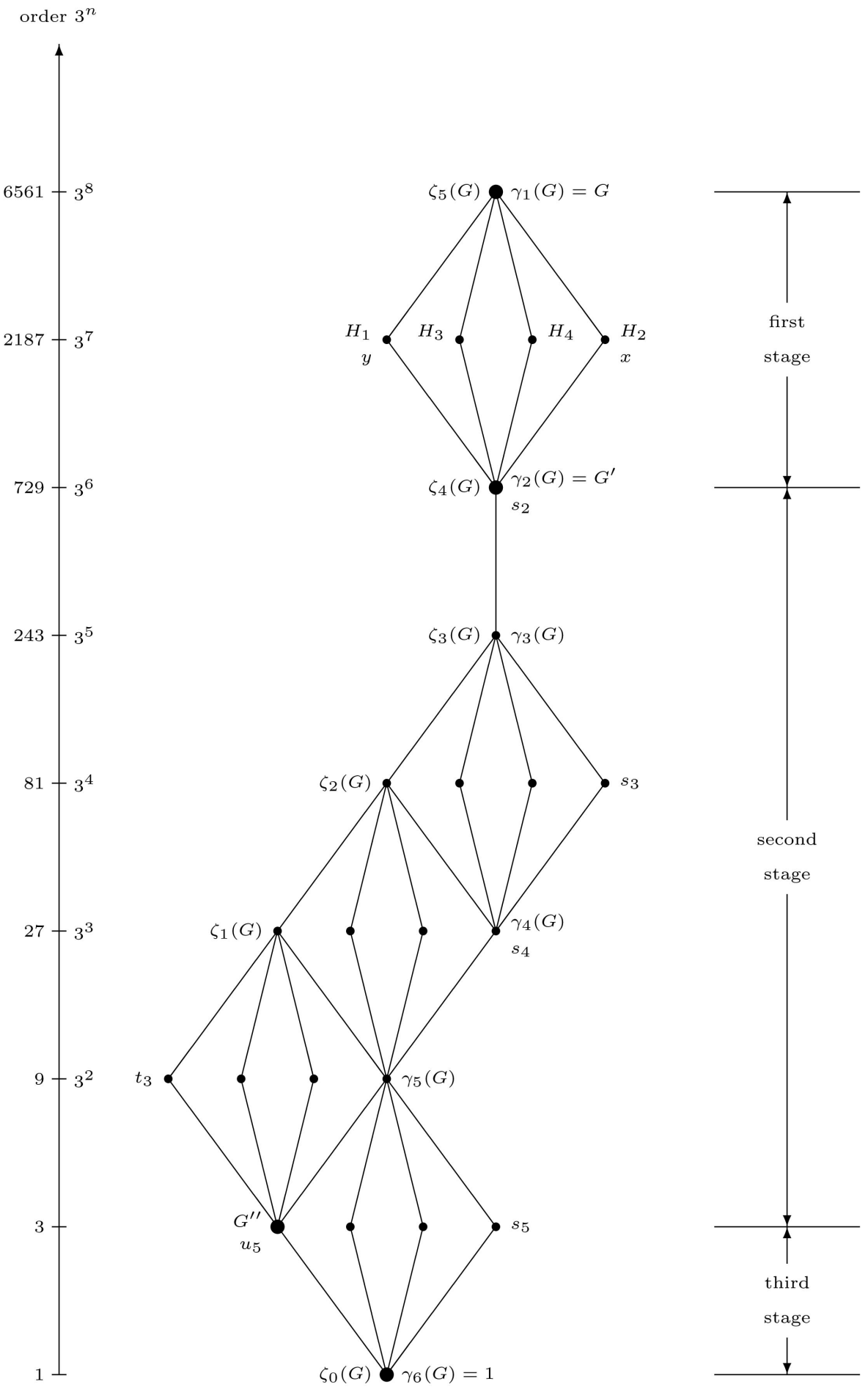

Figure 10. Normal Lattice and Central Series of $G_{3}^{5}(z, w)$.

2) their second derived group $G^{\prime \prime}<\zeta_{1}(G)$ is central and cyclic of order $3^{j+1}$.

Furthermore,

- they are Schur $\sigma$-groups with automorphism group $\operatorname{Aut}(G)$ of order $2 \cdot 3^{4 j+10}$,

- the factors of their lower central series are given by 


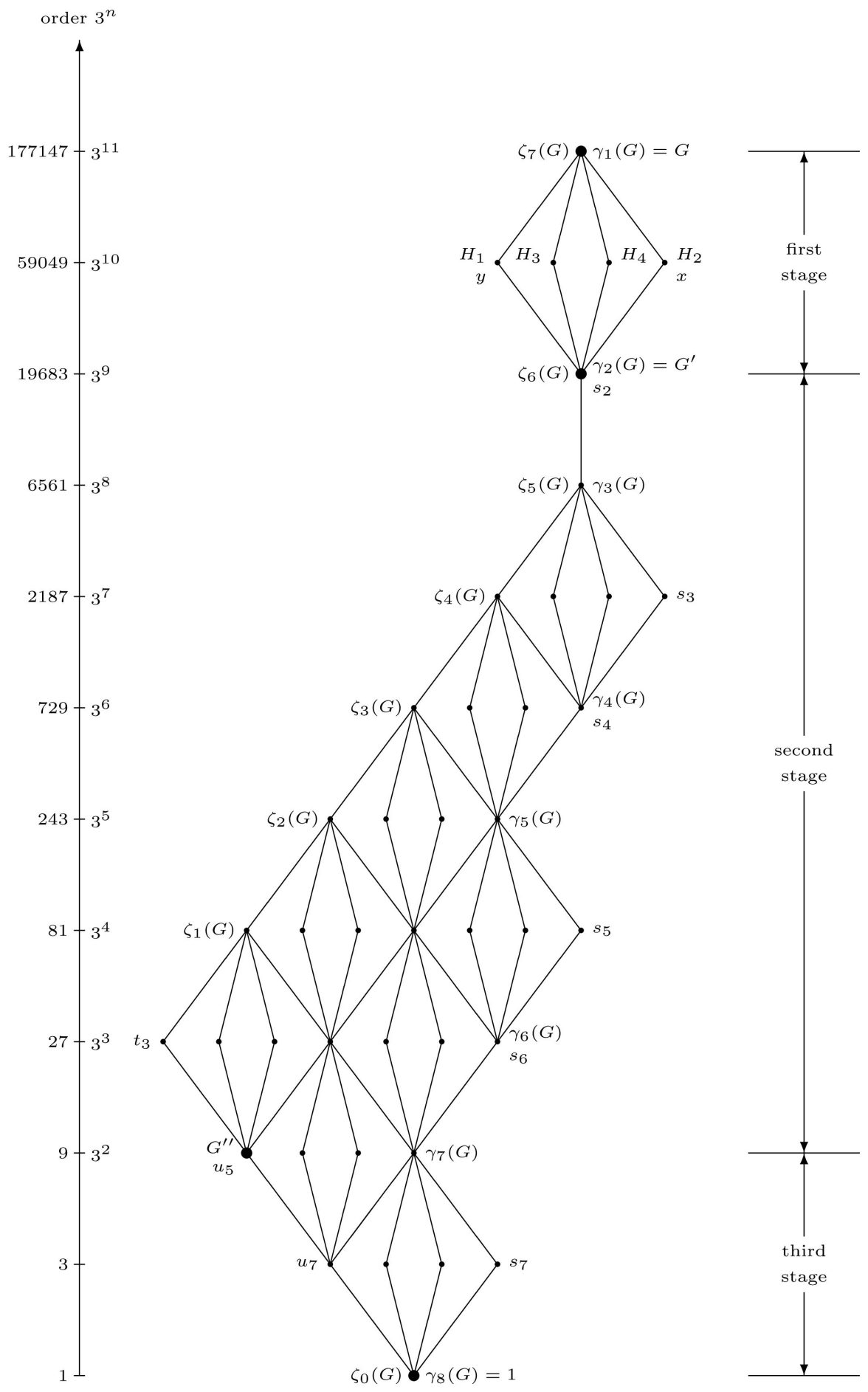

Figure 11. Normal Lattice and Central Series of $G_{4}^{7}(z, w)$.

$$
\gamma_{k}(G) / \gamma_{k+1}(G) \simeq \begin{cases}(3,3), & \text { for odd } 1 \leq k \leq 2 j+5, \\ (3), & \text { for even } 2 \leq k \leq 2 j+4,\end{cases}
$$

- their metabelianization $G / G^{\prime \prime}$ is of order $3^{2 j+7}$, class $2 j+5$ and of fixed coclass 2, 
- their biggest metabelian ancestor, that is the $(2 j+1)$ th iterated parent, is given by either $\langle 729,49\rangle$ or $\langle 729,54\rangle$.

\section{Conclusion}

We emphasize that the results of Section 21.2 provide the background for considerably stronger assertions than those made in [7] (which are, however, sufficient already to disprove erroneous claims in [48] [49]). Firstly, they concern four TKTs E.6, E.14, E.8 and E.9 instead of just TKT E.9, and secondly, they apply to varying odd nilpotency class $5 \leq \mathrm{cl}(G) \leq 19$ instead of just class 5 .

\section{Acknowledgements}

We gratefully acknowledge that our research is supported by the Austrian Science Fund (FWF): P 26008-N25. We are indebted to the anonymous referees for valuable suggestions improving the exposition and readability.

\section{References}

[1] du Sautoy, M. (2001) Counting p-Groups and Nilpotent Groups. Publications Mathématiques de l'Institut des Hautes Études Scientifiques, 92, 63-112. http://dx.doi.org/10.1007/BF02698914

[2] Eick, B. and Leedham-Green, C. (2008) On the Classification of Prime-Power Groups by Coclass. Bulletin of the London Mathematical Society, 40, 274-288. http://dx.doi.org/10.1112/blms/bdn007

[3] Artin, E. (1929) Idealklassen in Oberkörpern und allgemeines Reziprozitätsgesetz. Abhandlungen aus dem Mathematischen Seminar der Universität Hamburg, 7, 46-51. http://dx.doi.org/10.1007/BF02941159

[4] Mayer, D.C. (2012) Transfers of Metabelian p-Groups. Monatshefte für Mathematik, 166, 467-495. http://dx.doi.org/10.1007/s00605-010-0277-x

[5] Mayer, D.C. (2014) Principalization Algorithm via Class Group Structure. Journal de Théorie des Nombres de Bordeaux, 26, 415-464.

[6] Mayer, D.C. (2013) The Distribution of Second p-Class Groups on Coclass Graphs. Journal de Théorie des Nombres de Bordeaux, 25, 401-456.

[7] Bush, M.R. and Mayer, D.C. (2015) 3-Class Field Towers of Exact Length 3. Journal of Number Theory, 147, $766-777$. http://dx.doi.org/10.1016/j.jnt.2014.08.010

[8] Newman, M.F. (1977) Determination of Groups of Prime-Power Order. In: Bryce, R.A., Cossey, J., Newman, M.F., Eds., Group Theory, Springer, Berlin, 73-84.

[9] O’Brien, E.A. (1990) The p-Group Generation Algorithm. Journal of Symbolic Computation, 9, 677-698. http://dx.doi.org/10.1016/S0747-7171(08)80082-X

[10] Holt, D.F., Eick, B. and O’Brien, E.A. (2005) Handbook of Computational Group Theory, Discrete Mathematics and Its Applications. Chapman and Hall/CRC Press, Boca Raton. http://dx.doi.org/10.1201/9781420035216

[11] Gamble, G., Nickel, W. and O'Brien, E.A. (2006) ANU p-Quotient—p-Quotient and p-Group Generation Algorithms. An Accepted GAP 4 Package, Available also in MAGMA.

[12] The GAP Group (2014) GAP - Groups, Algorithms, and Programming - A System for Computational Discrete Algebra. Version 4.7.5, Aachen, Braunschweig, Fort Collins, St. Andrews. http://www.gap-system.org

[13] The MAGMA Group (2014) MAGMA Computational Algebra System. Version 2.21-1, Sydney. http://magma.maths.usyd.edu.au

[14] Ascione, J.A., Havas, G. and Leedham-Green, C.R. (1977) A Computer Aided Classification of Certain Groups of Prime Power Order. Bulletin of the Australian Mathematical Society, 17, 257-274. http://dx.doi.org/10.1017/s0004972700010467

[15] Nebelung, B. (1989) Klassifikation metabelscher 3-Gruppen mit Faktorkommutatorgruppe vom Typ $(3,3)$ und Anwendung auf das Kapitulationsproblem. Inaugural Dissertation, Universität zu Köln, Köln.

[16] Besche, H.U., Eick, B. and O’Brien, E.A. (2002) A Millennium Project: Constructing Small Groups. International Journal of Algebra and Computation, 12, 623-644. http://dx.doi.org/10.1142/S0218196702001115

[17] Besche, H.U., Eick, B. and O’Brien, E.A. (2005) The Small Groups Library-A Library of Groups of Small Order. An Accepted and Refereed GAP 4 Package, Available also in MAGMA.

[18] Ascione, J.A. (1979) On 3-Groups of Second Maximal Class. Ph.D. Thesis, Australian National University, Canberra.

[19] Ascione, J.A. (1980) On 3-Groups of Second Maximal Class. Bulletin of the Australian Mathematical Society, 21, $473-$ 
474. http://dx.doi.org/10.1017/S0004972700006298

[20] Newman, M.F. (1990) Groups of Prime-Power Order. Lecture Notes in Mathematics, 1456, 49-62. http://dx.doi.org/10.1007/BFb0100730

[21] Leedham-Green, C.R. and Newman, M.F. (1980) Space Groups and Groups of Prime Power Order I. Archiv der Mathematik, 35, 193-203. http://dx.doi.org/10.1007/BF01235338

[22] du Sautoy, M. and Segal, D. (2000) Zeta Functions of Groups. In: du Sautoy, M., Segal, D. and Shalev, A., Eds., New Horizons in Pro-p Groups, Progress in Mathematics, Birkhäuser, Basel, 249-286.

[23] Leedham-Green, C.R. and McKay, S. (2002) The Structure of Groups of Prime Power Order, London Mathematical Society Monographs. New Series 27, Oxford University Press, Oxford.

[24] Eick, B., Leedham-Green, C.R., Newman, M.F. and O’Brien, E.A. (2013) On the Classification of Groups of PrimePower Order by Coclass: The 3-Groups of Coclass 2. International Journal of Algebra and Computation, 23, 12431288. http://dx.doi.org/10.1142/s0218196713500252

[25] Newman, M.F. and O’Brien, E.A. (1999) Classifying 2-Groups by Coclass. Transactions of the American Mathematical Society, 351, 131-169. http://dx.doi.org/10.1090/S0002-9947-99-02124-8

[26] Dietrich, H., Eick, B. and Feichtenschlager, D. (2008) Investigating $p$-Groups by Coclass with GAP. In: Kappe, L.-C., Magidin, A. and Morse, R.F., Eds., Computational Group Theory and the Theory of Groups, AMS, Providence, 45-61. http://dx.doi.org/10.1090/conm/470/09185

[27] Shalev, A. (1994) The Structure of Finite $p$-Groups: Effective Proof of the Coclass Conjectures. Inventiones Mathematicae, 115, 315-345. http://dx.doi.org/10.1007/BF01231763

[28] Leedham-Green, C.R. (1994) The Structure of Finite p-Groups. Journal London Mathematical Society, 50, $49-67$. http://dx.doi.org/10.1112/jlms/50.1.49

[29] Hall, M. and Senior, J.K. (1964) The Groups of Order $2^{\mathrm{n}}(\mathrm{n} \leq 6)$. Macmillan, New York.

[30] Hall, P. (1940) The Classification of Prime-Power Groups. Journal für Die Reine und Angewandte Mathematik, 182, 130-141.

[31] Blackburn, N. (1958) On a Special Class of p-Groups. Acta Mathematica, 100, 45-92. http://dx.doi.org/10.1007/BF02559602

[32] Taussky, O. (1937) A Remark on the Class Field Tower. Journal London Mathematical Society, 12, 82-85. http://dx.doi.org/10.1112/jlms/s1-12.1.82

[33] Bagnera, G. (1898) La composizione dei gruppi finiti il cui grado è la quinta potenza di un numero primo. Annali di Matematica Pura ed Applicata, 1, 137-228. http://dx.doi.org/10.1007/bf02419191

[34] Arrigoni, M. (1998) On Schur $\sigma$-Groups. Mathematische Nachrichten, 192, 71-89. http://dx.doi.org/10.1002/mana.19981920105

[35] Boston, N., Bush, M.R. and Hajir, F. (2015) Heuristics for $p$-Class Towers of Imaginary Quadratic Fields. Mathematische Annalen, in Press.

[36] Koch, H. and Venkov, B.B. (1975) Über den p-Klassenkörperturm eines imaginär-quadratischen Zahlkörpers. Astérisque, 24-25, 57-67.

[37] Benjamin, E., Lemmermeyer, F. and Snyder, C. (2003) Imaginary Quadratic Fields with $\mathrm{Cl}_{2}(k) \simeq(2,2,2)$. Journal of Number Theory, 103, 38-70. http://dx.doi.org/10.1016/S0022-314X(03)00084-2

[38] Shafarevich, I.R. (1964) Extensions with Prescribed Ramification Points (Russian). Publications Mathématiques de l'IHÉS, 18, 71-95.

[39] Boston, N. and Nover, H. (2006) Computing Pro-p Galois Groups. Algorithmic Number Theory: Lecture Notes in Computer Science, 4076, 1-10.

[40] Mayer, D.C. (2012) The Second p-Class Group of a Number Field. International Journal of Number Theory, 8, 471505. http://dx.doi.org/10.1142/S179304211250025X

[41] Nover, H. (2009) Computation of Galois Groups of 2-Class Towers. Ph.D. Thesis, University of Wisconsin, Madison.

[42] Azizi, A., Zekhnini, A. and Taous, M. (2015) Coclass of $\operatorname{Gal}\left(k_{2}^{(2)} \mid k\right)$ for Some Fields $k=\mathbb{Q}\left(\sqrt{p_{1} p_{2} q}, \sqrt{-1}\right)$ with 2-Class Groups of Type (2,2,2). Journal of Algebra and Its Applications, in Press.

[43] Bosma, W., Cannon, J. and Playoust, C. (1997) The Magma Algebra System. I. The User Language. Journal of Symbolic Computation, 24, 235-265. http://dx.doi.org/10.1006/jsco.1996.0125

[44] Bosma, W., Cannon, J.J., Fieker, C. and Steels, A., Eds. (2014) Handbook of Magma Functions. Edition 2.21, University of Sydney, Sydney. 
[45] Mayer, D.C. and Newman, M.F. (2013) Finite 3-Groups as Viewed from Class Field Theory, Groups St. Andrews 2013. University of St. Andrews, Fife, Scotland.

[46] Mayer, D.C., Bush, M.R. and Newman, M.F. (2013) 3-Class Field Towers of Exact Length 3. 18th ÖMG Congress and 123rd Annual DMV Meeting 2013, University of Innsbruck, Tyrol, Austria.

[47] Mayer, D.C., Bush, M.R. and Newman, M.F. (2013) Class Towers and Capitulation over Quadratic Fields. West Coast Number Theory 2013, Asilomar Conference Center, Pacific Grove.

[48] Scholz, A. and Taussky, O. (1934) Die Hauptideale der kubischen Klassenkörper imaginär quadratischer Zahlkörper: Ihre rechnerische Bestimmung und ihr Einfluß auf den Klassenkörperturm. Journal Für Die Reine und Angewandte Mathematik, 171, 19-41.

[49] Heider, F.-P. and Schmithals, B. (1982) Zur Kapitulation der Idealklassen in unverzweigten primzyklischen Erweiterungen. Journal Für Die Reine und Angewandte Mathematik, 336, 1-25.

[50] Mayer, D.C. (1991) Principalization in Complex $S_{3}$-Fields. Congressus Numerantium, 80, 73-87. 